\title{
Theoretischer Rahmen
}

Die für diese Studie relevanten theoretischen Konzepte und Modelle haben sich im Laufe des zirkulären Forschungsprogramms der Grounded Theory aus dem empirischen Material sowie aus den Untersuchungen, Ableitungen und Interpretationen ergeben. In einer steten Bewegung zwischen Empirie und Theorie sind erst nach einigen Interpretationsansätzen relevante theoretische Konzepte entstanden, die kombiniert und auf je unterschiedliche Art und Weise auf die einzelnen Fallbeispiele angewendet werden. Beabsichtigt ist eine die Empirie anregende Heuristik (Reckwitz 2015: 28), welche die Kontexte der Fallstudien sichtbar machen soll. Die folgenden Ausführungen und Bestimmungen werden bewusst kurz gehalten, um den Rahmen der Arbeit nicht unnötig zu sprengen. Sie sollen das Handwerkszeug aufzeigen, mit dem die Fallstudien und -analysen nachzuvollziehen sind.

Zunächst wird das Konzept der „totalen Institution“ aus Erving Goffmans Studie (1973) vorgestellt (Abschn. 4.1). Anschließend werden die Begriffe Affekt und Emotion erläutert und darauf aufbauend ein Multikomponentensystem als Emotionsmodell präsentiert (Abschn. 4.2.1. und 4.2.2). Danach werden zwei Ansätze der Emotionsforschung diskutiert, zum einen die Emotionstheorie von Jack Katz (Abschn. 4.2.3) und zum anderen das Konzept der existenziellen Gefühle von Matthew Ratcliffe (Abschn. 4.2.4). Darauf folgt eine Erläuterung des Postkolonialismus (Abschn. 4.3) und die Diskussion der Konzepte von Gayatri Chakravorty Spivak (Abschn. 4.4) sowie Homi K. Bhabha und seiner Hauptbegriffe (Abschn. 4.5). Im folgenden Unterkapitel wird mit Judith Butlers Theorie der Subjektivation die Subjektwerdung unter postmodernen Bedingungen und damit der Prozess der Unterwerfung beleuchtet (Abschn. 4.6). Abgeschlossen wird das Theoriekapitel mit einer Explikation der Herr-Knecht-Figur von Hegel (Abschn. 4.7). 


\subsection{Erving Goffman: Totale Institution}

Der kanadische Soziologe Erving Goffman (1922-1982) hinterließ ein umfangreiches Werk und beeinflusste die Soziologie nachhaltig mit seinen innovativen Ansätzen und Ideen. Nach seinen Studien in Toronto und an der Universität von Chicago führte er seine Feldforschung (1949-1951) auf den Shettland-Inseln Schottlands durch und promovierte anschließend mit der daraus entstandenen Dissertation bei Anselm Strauss. Daraufhin begannen seine eigenständigen Studien, unter anderem in Anstalten (Asyle 1961), bis er 1962 in Berkeley und 1969 in Pennsylvania eine Professur annahm (Knoblauch 2006: 157).

Zentral für die Ausarbeitung seiner Ansätze ist die (sozial-)phänomenologische Richtung, die sich auf Alfred Schütz (1899-1959) und Edmund Husserl (1859-1938) beruft (Hettlage 2008: 253). Die Soziologie wurde erst nach seinem Tod auf seine Untersuchungen aufmerksam, obwohl seine Werke zu Lebzeiten auch außerhalb der Wissenschaft rezipiert wurden (Bergner 2010: 8). Der Grund für den lange ausbleibenden Erfolg innerhalb der wissenschaftlichen Disziplin liegt wohl vor allem darin, dass Goffman keine „große soziologische Theorie“ formulierte (Hettlage/Lenz 1991: 16). Vielmehr etablierte er eine Art und Weise der Forschung, die sich deutlich von den bisherigen sozialwissenschaftlichen Standards unterschied, vor allem indem er den sozialen Alltag mit seinen Überraschungsmomenten humorvoll und in leicht verständlicher Form analysierte (ebd.: 189). Seine herausragende Bedeutung zeigt sich etwa darin, dass seine Werke weit über die Grenzen der Soziologie hinaus - auch außerhalb der Wissenschaft - mit großer Aufmerksamkeit rezipiert worden sind (Engelhardt 2010: 123).Das Goffman'sche Werk lässt sich schwerlich anhand einer bestimmten Folie lesen und verstehen (Raab 2008: 9). Dessen ungeachtet stehen, unabhängig vom Forschungsfeld, bestimmte Themenkomplexe im Vordergrund seines Interesses, etwa die verschiedenen Dimensionen der Selbstdarstellung und deren Einbettung in soziale Interaktionen (Goffman; 1983 [1959], 1973), die Rahmenanalyse (1980) sowie seine Untersuchungen zu „Rede-Weisen“ (vgl. Knoblauch et al. 2005; Keller 2012: 285). Dabei spielen die Begriffe Rahmenanalyse und Interaktionsordnung eine maßgebliche Rolle für das Verständnis seines Werks. Goffman selbst bezeichnete kurz vor seinem frühen Tod in seiner geplanten Ansprache vor der American Sociological Association die Interaktionsordnung als den genuinen Gegenstand seines Werks (Goffmann 1983a). Diese sei weniger eine Theorie als vielmehr ein Forschungsprogramm. Die Ordnung sozialer Interaktionen oder, genauer gesagt, die unmittelbare soziale Interaktion im Zwischenmenschlichen, bei der unterschiedliche Individuen versuchen, eine 
gemeinsame Wirklichkeit herzustellen (Engelhardt 2010: 123), kann demzufolge als Goffmans zentrales Forschungsinteresse betrachtet werden. Von Bedeutung ist hierbei stets die Frage, wie soziale Situationen strukturiert sind. Goffman geht es „,nicht um Menschen und ihre Situationen“, sondern um „Situationen und ihre Menschen“ (Keller 2012: 290).

\section{Das Konzept der totalen Institution}

Der Begriff der totalen Institution hat sich innerhalb der Wissenschaft rasch etabliert (Eisenbach-Stangl 1978: 4), jedoch fehlt aufgrund seines vermeintlichen Allgemeinverständnisses eine tiefgehende Betrachtung (Bammann 2010: 10). Dies liegt vor allem daran, dass sich der Begriff meist auf die räumlichen Institutionen der Psychiatrie sowie des Strafvollzuges bezieht und nicht über diese Einrichtungen hinaus auf andere Lebensbereiche angewendet wird. So findet er sich auch bei Michel Foucault, jedoch geht es dabei im Unterschied zu Goffman um die Geschichte und um historische Deduktionen moderner Gesellschaftsprozesse (ebd.: 38). Anders als bei Goffman liegt bei Foucault der Fokus zudem auf Prozessen und Mechanismen der Machtproduktion hinter der totalen Institution. Bemerkenswerterweise leitet Foucault in seiner 1975 erschienen Abhandlung Überwachen und Strafen (1994) den Begriff nicht von Goffman, sondern von Louis-Pierre Baltard ${ }^{1}$ ab (ebd.: 301) und erwähnt Goffman kein einziges $\mathrm{Mal}^{2}$. Es bleibt in der Literatur oft bei einem bloßen Verweis auf Goffmans Asyle oder einer sehr kurzen Darstellung seiner Aussagen, ohne das dahinterstehende Konzept auf weitere Felder zu beziehen (vgl. Feest et al. 1997; Bögemann 2004), oder aber es kommt zu einer inflationären Verwendung des Begriffs der totalen Institution, so dass sich die Frage aufdrängt, ob der Gebrauch Goffman'scher Termini überhaupt angemessen ist (z. B. Davies 1989; Liniany 2008). Täubig (2009) bezieht ihn erstmals systematisch auf das Leben von Asylbewerber*innen, wobei sie nicht bloß die rein physischen Unterkünfte als totale Institutionen auffasst, sondern überhaupt den Status Geflüchteter und deren alltägliche Art und Weise des Umgangs mit widrigen Situationen. Goffman veröffentlichte 1961 seine Studie Asyle. Über die soziale Situation psychiatrischer Patienten und anderer Insassen. ${ }^{3}$ Mit seiner Forschung gibt er Einblicke in das

\footnotetext{
${ }^{1}$ Er bezieht sich dabei auf dessen 1829 erschienene Abhandlung Architectonographie des prisons.

${ }^{2}$ Anthony Giddens (1988: 199-214) unternimmt einen handlungstheoretischen Vergleich zwischen den unterschiedlichen Konzepten Goffmans und Foucaults.

${ }^{3}$ Deutsche Fassung 1973.
} 
Innenleben einer psychiatrischen Anstalt und entwickelt einen neuartigen Bezugsrahmen für die Erfassung komplexer sozialer Gebilde. Für dieses Konzept prägt er anhand des Beispiels einer psychiatrischen Klinik den Begriff der „Totalen Institution“ (Heinzelmann 2004: 54). Unter Institutionen versteht er zunächst Räume, in denen regelmäßig eine bestimmte Tätigkeit ausgeübt wird, wobei auch explizit „Wohnungen, Gebäude oder Betriebe“ zu den Räumen hinzugezählt werden (Goffman 1973: 15). Der allumfassende oder totale Charakter einer Institution ergibt sich durch die Beschränkungen der Mitglieder, mit der Außenwelt zu interagieren. Er fasst somit unterschiedliche Einrichtungstypen wie etwa Klöster, Gefängnisse, Psychiatrien, Kasernen und Konzentrationslager zusammen. Der Begriff der totalen Institution deutet an, dass diese Einrichtungen prinzipiell umfassender sind als andere: Sie beanspruchen erheblich mehr Zeit der Insassen. Im Vordergrund stehen der Zwangscharakter gesellschaftlicher Institutionen und die Möglichkeit der Identitätsbildung, die sich aus dem Zwang ergibt. In den vier Essays aus Asyle beschreibt Goffman die geschlossene Welt hinter dem Organisationskomplex der Psychiatrie, in der er seine ethnografische Studie durchführte (Hettlage 2008: 255). Alle Aktivitäten finden für die Dauer ihres Aufenthaltes in derselben Einrichtung statt. Unter dem Begriff der totalen Institution versteht Goffman konkret:

„Eine totale Institution lässt sich als Wohn- und Arbeitsstätte einer Vielzahl ähnlich gestellter Individuen definieren, die für längere Zeit von der übrigen Gesellschaft abgeschnitten sind und miteinander ein abgeschlossenes, formal reglementiertes Leben führen. Ein anschauliches Beispiel dafür sind Gefängnisse, vorausgesetzt, dass wir zugeben, dass das, was an Gefängnissen gefängnisartig ist, sich auch in anderen Institutionen findet, deren Mitglieder keine Gesetze übertreten haben“ (1973: 11).

Totale Institutionen verknüpfen die beiden Bereiche des privaten und des beruflichen Lebens miteinander. Die gewöhnliche Trennung zwischen Zuhause und Arbeit sowie der Heim- bzw. Arbeitsweg entfallen hier (Bammann 2010: 16). Goffmans totale Institution befindet sich somit nicht auf der abstrakten Ebene, sondern ist real bestimmbar und lässt sich durch „verschlossene Tore, hohe Mauern, Stacheldraht, Felsen, Wasser, Wälder oder Moore" (Goffman 1973: 15) begrenzen. Dass es sich um einen realen und nicht abstrakten Ort handelt, deutet bereits der Titel Asyle (Asylums) an, ein Zufluchtsort für Menschen also, 
die außerhalb der Institution nicht zurechtkommen würden. ${ }^{4}$ Die normale soziale Ordnung der modernen Gesellschaft, die sich dadurch kennzeichnet, dass ,der einzelne an verschiedenen Orten schläft, spielt, arbeitet - und dies mit wechselnden Partnern, unter verschiedenen Autoritäten und ohne einen umfassenden rationalen Plan“ (ebd.: 17), wird durch die totale Institution aufgehoben. Die Trennung der genannten drei Bereiche ist das zentrale Merkmal. Goffman unterscheidet insgesamt fünf Gruppen totaler Institutionen:

I) Einrichtungen der Fürsorge für hilflose Menschen (z. B. Altersheime, Blindenheime, Armen- und Waisenhäuser)

II) Einrichtungen der Fürsorge für hilflose Menschen, vor denen die Öffentlichkeit zu schützen ist (z. B. Irrenhäuser, Tuberkulose-Sanatorien und Leprosorien)

III) Einrichtungen, die dem Schutz der Gesellschaft vor Gefahren durch die Insassen dienen (z. B. Gefängnisse, Zuchthäuser, Konzentrationslager, Kriegsgefangenenlager)

IV) Einrichtungen, die eine verbesserte Arbeitsorganisation gewährleisten sollen (z. B. Kasernen, Schiffe, Internate, Arbeitslager, koloniale Stützpunkte und Gutshäuser)

V) Einrichtungen, die einen Rückzug aus der Welt ermöglichen (z. B. Abteien, Klöster und Konvente)

Zugleich verweist Goffman auf die Gemeinsamkeiten der unterschiedlichen Beispiele totaler Institutionen. Diese Vermengung mag kritisch gesehen werden, da die Ziele einzelner Institutionen grundverschieden sind. Andererseits können trotz verschiedener Zielsetzungen die Voraussetzungen und Konsequenzen ähnlich sein, wie etwa Siegler und Osmond (1971: 419 f.) zu zeigen versucht haben. Goffman begründet den Zusammenhang anhand der Methoden und Prozesse, durch die totale Institutionen ihre Mitglieder demütigen (Bammann 2010: 27). Als gemeinsame Attribute der verschiedenen Arten totaler Institutionen führt er an (Goffman 1973: 17):

a) Sämtliche Angelegenheiten des Lebens finden an derselben Stelle und unter derselben Autorität statt.

\footnotetext{
${ }^{4}$ Einige verstehen im Übrigen den Begriff „Asyl“ hierbei durchaus ironisch (Scheutz 2008: 4), da Asyle eben genau jene Effekte hervorrufen können, vor denen das Asyl ihre Mitglieder beschützen soll (Bammann 2010: 19).
} 
b) Die Mitglieder der Einrichtungen führen alle Phasen ihrer täglichen Arbeit in unmittelbarer Gesellschaft einer großen Gruppe von Schicksalsgenossen aus, wobei allen die gleiche Behandlung zuteil wird und alle die gleiche Tätigkeit gemeinsam verrichten müssen.

c) Alle Phasen des Arbeitstages sind exakt geplant, die eine geht zu einem vorher bestimmten Zeitpunkt in die nächste über und die ganze Folge der Tätigkeiten wird von oben durch ein System expliziter formaler Regeln und durch einen Stab von Funktionären zugeschrieben.

d) Die verschiedenen erzwungenen Tätigkeiten werden in einem einzigen rationalen Plan vereinigt, der vorgeblich dazu dient, die offiziellen Ziele der Institution zu erreichen.

Als zentrales Faktum totaler Institutionen stellt Goffman den Umgang mit menschlichen Bedürfnissen durch die bürokratische Organisation fest (1973: 18). Wenn etwa Insassen in „Blöcken bewegt werden“ (ebd.), so schlussfolgert Goffman, richtet sich die Aufmerksamkeit des Personals nicht mehr auf die Führung, sondern auf die Überwachung der gefangenen Menschen. Im Konzept der totalen Institution besteht daher eine grundlegende Trennung zwischen einer großen Menschengruppe, den Insassen, und einer viel kleineren Gruppe, dem Aufsichtspersonal, die erstere verwaltet (ebd.). Cox (1978: 46) spricht in diesem Punkt daher von Opfern und nicht von Insassen. Die negativen, teils psychiatrisch relevanten Konsequenzen für die gefangenen Menschen werden in Goffmans Buch häufig erwähnt. Im Prinzip können weite Teile seiner Studie Asyle als eine systematische Untersuchung negativer psychischer und psychiatrischer Folgen eines Aufenthalts in totalen Institutionen verstanden werden. Für das gefangene Subjekt ergibt sich aus diesen Dynamiken ein sozialer bzw. „bürgerlicher Tod“ (1973: 26).

\section{Der bürgerliche Tod}

Gefangene werden in einer Demütigungsphase zu Beginn ihrer ehemaligen Identität beraubt. Von der Anstaltskleidung bis über die fehlende zeitliche und räumliche Privatsphäre wird das Subjekt systematisch seines bisherigen Selbstverständnisses, entkleidet‘.

„Neuankömmlinge kommen mit einem bestimmten Bild von sich selbst in die Anstalt, welches durch bestimmte stabile soziale Bedingungen seiner heimischen Umgebung ermöglicht wurde. Beim Eintritt wird er sofort der Hilfe beraubt, die diese Bedingungen ihm boten. In der exakten Sprache einer unserer ältesten totalen Institutionen durchläuft er eine Reihe von Erniedrigungen, Degradierungen, 
Demütigungen und Entwürdigungen seines Ich. Sein Ich wird systematisch, wenn auch häufig unbeabsichtigt, gedemütigt. Es treten einige radikale Veränderungen seiner moralischen Laufbahn ein, einer Laufbahn, die sich aus den progressiven Veränderungen zusammensetzt, die in den Überzeugungen stattfinden, die er über sich und andere wichtige Personen hegt" (Goffman 1973: 25).

Persönliche Informationen werden öffentlich gemacht; zugleich bleibt das gefangene Subjekt innerhalb der geschlossenen Institution sich selbst überlassen, so dass keine Distanz zwischen dem Selbst und entwürdigenden Situationen aufgebaut werden kann (Hettlage 2008: 256). Die Prozesse, durch die das Ich eines Menschen gedemütigt wird, ähneln sich im Regelfall. Ihre Analyse kann helfen, die Bedingungen $\mathrm{zu}$ erkennen, die konventionelle Institutionen garantieren müssen, damit ihre Insassen nicht sozial sterben (ebd.). Der Aufenthalt in einer totalen Institution beginnt mit spezifischen Aufnahmeritualen, wie der Aufnahme des Lebenslaufes, Fotografieren, Messen, Wiegen, Abnehmen der Fingerabdrücke, Leibesvisitation, Einweisung in die Hausordnung usw. Der Eintritt in die Institution kommt deshalb dem bürgerlichen Tod des Insassen gleich, weil die Zugehörigkeit zur totalen Institution die planmäßige Rolle des Individuums und damit auch sämtliche sozialen und persönlichen Verbindungen mit einem Schlag unterbricht (ebd.). In totalen Institutionen kommen Menschen notgedrungen zusammen und verbinden sich zu Schicksalsgemeinschaften. So entstehen neue soziale Beziehungen und Dynamiken, die mehr und mehr zur Normalität werden. Dabei halten die Insassen die Bediensteten für herablassend, hochmütig und niederträchtig, während Letztere die Ersteren für verbittert, verschlossen und wenig vertrauenswürdig halten (ebd.: 19). Da das Personal standardmäßig glaubt, das Recht auf seiner Seite zu haben, gelten ihm die Insassen als unterlegen und schwach. Dies äußert sich in der grundlegenden sozialen Asymmetrie und Distanz zwischen beiden Gruppen, an der sich neu ankommende Insassen in der Regel orientieren (ebd.: 61). Sobald die Demütigungen und die Angriffe auf das Selbst in Form der Kontrolle des persönlichen Raums und der persönlichen Zeit kontinuierlich ausgeführt werden, beginnt die systematische Persönlichkeitsveränderung, etwa durch die Vergabe von Privilegien in Form von Tabak, Kaffee, Toilettenpapier und Süßigkeiten. Individuelles Verhalten wird sozial belohnt oder sanktioniert (Malacrida 2005: 531). Zentral für den Rollen- und Identitätsverlust ist nicht nur die neue fundamentale Asymmetrie im Sozialen, sondern die Ausgesetztheit an ein Leben ohne das Recht, monetäre Mittel zu besitzen, Ehescheidungen oder Adoptionen zu beantragen und zu wählen (ebd.: 26). Goffman zeichnet eine "Schranke“ (ebd.: 25) nach, die in totalen Institutionen zwischen Insassen und Personal besteht und die durch ihr Wirken fortwährend reproduziert 
wird. Die unterlegene Gruppe wird in diesem Zuge von der Gesellschaft ausschlossen, was wiederum dazu führt, dass jene schwer zu erlangenden Elemente, wie etwa Kino, Fernsehen, Lesen, Alkohol und Tabak (ebd.: 45), mithilfe derer sie das „Gefühl der toten und bleischweren Zeit“ (ebd.: 72) zu überwinden sucht, $\mathrm{zu}$ Vergünstigungen werden. Ein weiteres Kennzeichen des bürgerlichen Todes innerhalb totaler Institutionen ist die enorme Zeitvergeudung (ebd.: 71). Die Insassen vieler totaler Institutionen haben das Gefühl, dass die in der Anstalt verbrachte Zeit vergeudete Zeit ist:

„In Gefängnissen und Heilanstalten wird der Eingewöhnungserfolg im allgemeinen danach gewertet, wie der Insasse mit dem Verstreichen der Zeit zurechtkommt, ob es ihm schwer oder leicht fällt. Diese Zeit wird von denjenigen, die sie absitzen müssen, bewußt ausgeklammert, und sie beschäftigen sich in Gedanken dauernd mit ihr, in einem Maße, wie dies draußen nicht üblich ist. Folglich bekommt der Insasse das Gefühl, daß er für die Dauer seines Aufenthaltes - seiner Haft - vollkommen vom Leben ausgeschlossen ist“" (ebd.).

Gefangene bauen sich durch sogenannte „sekundäre Anpassungsmechanismen“ Gegenwelten innerhalb der Institution auf, um strategisch gegen die Totalität der Kontrolle vorzugehen (ebd.: 252). Diese Anpassungsmechanismen dienen dem Selbstschutz der Gefangenen, doch resultieren sie darin, dass sie die anfängliche Diagnose bestätigen. Jeglicher Versuch des gefangenen Individuums, seine Würde zu wahren und gegen die Totalität zu rebellieren, wird seitens des Personals als Bestätigung des Symptoms der Krankheit begriffen, das ursprünglich zum Eintritt der betroffenen Person in die Institution geführt hat (Hettlage 2008: 257). Mit seiner Studie zeigt Goffman vor allem die Grenzen der Handlungsmächtigkeit eines Subjekts in totalen Institutionen auf. Die Selbstständigkeit des Subjekts kann sich angesichts eines so starr gezogenen Rahmens nicht entfalten, ohne einem strikten Determinismus zu verfallen. Durch Anpassungsmechanismen oder „Widerstandsformen“ etwa werden vielmehr „Räume dazwischen“ präsentiert, in denen das Subjekt seinen eigenen Bewegungsraum innerhalb einer Organisationsgesellschaft ergründet.

\subsection{Affekt und Emotion}

Melissa Gregg und Gregory J. Seigworth heben in ihrer Einleitung zum Affect Theory Reader hervor, dass ein Überblick über die breit gefächerten Ausdifferenzierungen der seit den 1990ern erfolgten affect studies unmöglich scheint (2010a). Seitdem entwickelte sich kontinuierlich eine weitreichende theoretische 
Debatte um das Verständnis und den Sinn von Affekten als Triebfedern menschlichen Handelns unter dem Oberbegriff „,affective turn“ (Clough \& Halley 2007). Die von Eva Illouz (2006) diagnostizierte Wende zum homo sentimentalis geht über eine bloße Berücksichtigung des Gefühlslebens innerhalb der Wissenschaften hinaus (Wiesse/Pfaller 2018: 3). Die diskursive Hinwendung zu Affekten in Gesellschaften und die programmatische Forderung nach affektiver Reflexivität rücken den sozialen Raum $^{5}$, die in ihm ausgeführten Praktiken und die prinzipielle Rolle von Affekten in den Fokus. Die sich häufenden Affektstudien und das Interesse an Affekten und Emotionen im Sozialen lassen dabei allerdings ein Desiderat an empirischen Untersuchungen zu Affekten erkennen (ebd.). Der turn to affect impliziert einen inhaltlichen Fokus auf diffuse, allgegenwärtige, stetig wirksame, körperlich ausgedrückte affektive Dynamiken, die menschliches Handeln, die zwischenmenschliche Interaktion und die Interaktion zwischen Menschen und Objekten motivieren (Lünenborg et al. 2018: 2). Affekttheoretische Ansätze finden in vielen wissenschaftlichen Disziplinen Anwendung, besonders weil sie neue Sichtweisen auf die Gestaltung gesellschaftlicher Ungleichheitsverhältnisse ermöglichen (ebd.). Gemeinsam haben alle Ansätze, dass sie Gefühle - ganz gleich, ob sie als Affekte oder als Emotionen bezeichnet werden - als soziokulturelle Praktiken verstehen, die maßgeblich auf Dynamiken sozialer Ordnungen einwirken und gleichsam durch soziale Ordnungen gerahmt werden. Eine Grundannahme bei der Auseinandersetzung mit Affekten besteht darin, dass sie als soziale Konstrukte zu sehen sind (Scheve/ Berg 2018: 27). Affekte treten demgemäß nicht vollkommen willkürlich und individuell in Erscheinung, sondern vielmehr systematisch, wobei insbesondere die strukturbildenden Faktoren von Machtverhältnissen, Werten, Normen und sozialen Netzwerken einbezogen werden. Ein Hauptanliegen der gegenwärtigen Emotionsforschung besteht daher darin, zu beleuchten, auf welche Weise Affekte und Emotionen von sozialen und kulturellen Gesichtspunkten modelliert werden.

Affekte werden grundsätzlich als „Hauptzutat“ oder Kern einer Emotion aufgefasst, doch die Heterogenität des Affektverständnisses selbst innerhalb der affect studies ist unübersehbar (vgl. etwa Gregg/Seigworth 2010; Leys 2011; Wetherell 2012). Die vorliegende Studie orientiert sich primär an den von der Berliner Schule ${ }^{6}$ entwickelten Kernkonzepten zur Affektivität (Slaby/Scheve

\footnotetext{
${ }^{5}$ Der bereits von der jungen Soziologie behandelt wurde (vgl. Georg Simmel 1995; Park/ Burgess 1984).

${ }^{6}$ Dies bezieht sich auf aus dem Sonderforschungsbereich 1171 Affective Societies Dynamiken des Zusammenlebens in bewegten Welten hervorgegangene Affektkonzepte der Freien Universität Berlin.
} 
2019), deren Grundaussagen sich sowohl in dem vorliegenden Emotionsmodell als auch in den gewählten Theoriesträngen widerspiegeln. Affekte stellen aus dieser Sicht eine unbestimmte und dynamische Größe zwischen Körpern dar und sind soziokulturell nicht kategorisiert bzw. in diskursiv etablierte Bahnen gelenkt worden (Röttger-Rössler 2016: 6). Sie werden als autonome Kräfte zwischen Körpern begriffen, die sich nicht in Emotionen und Gefühlen, sondern in „Intensitäten“ (Massumi 1995: 88) ausdrücken (Scheve/Berg 2018: 31). Diese zeigen an, inwiefern ein Körper selbst affiziert wird bzw. andere affizieren kann (Slaby/Mühlhoff 2019: 31; Clough 2007: 2). Affekte entziehen sich der reflektierenden Repräsentation, das heißt, sie befinden sich nicht bereits in einem eng gesteckten, von Bedeutungs- und Ausdruckskonventionen durchstrukturierten gesellschaftlichen Rahmen, der determiniert, auf welche spezifische Art und Weise Affekte wahrgenommen und ausgedrückt werden müssen (ebd.). Dennoch sind sie nicht völlig losgelöst von Sozialisations- und Enkulturationsprozessen. Sie entstehen etwa oft als gefühlte Differenz zu bereits Bestehendem (RöttgerRössler 2016: 6) - zum Beispiel dann, wenn Asylbewerber*innen im Ankunftskontext ihrer Flucht merken, dass sie gewisse soziale Eigentümlichkeiten oder Verhaltensweisen nicht verstehen. Ein Affekt

„is best understood as dynamic, intensive relations that unfold between human actors, in and with complex environmental settings, material formations, (urban) landscapes and designed spaces, various artifacts, technologies and media“" (Slaby/ Röttger-Rössler 2018: 3).

Der leibliche Aspekt kommt in Greggs und Seigworths Definition ebenfalls sehr klar zum Ausdruck:

\begin{abstract}
„Affect arises in the midst of in-between-ness: in the capacities to act and be acted upon. Affect is an impingement or extrusion of a momentary or sometimes more sustained state of relations as well as the passages (and the duration of passage) of forces or intensities. That is, affect is found in those intensities that pass body to body (human, non-human, part-body and otherwise), in those resonances that circulate about, between and sometimes stick to bodies and worlds, and in the very passages or variations between these intensities and resonances themselves" (Gregg/ Seigworth 2010: 1).
\end{abstract}

Das transpersonale Moment des affektiven Erlebens wurzelt philosophisch in der Affektenlehre Baruch de Spinozas (1632-1677), der als Gründervater einer Linie angesehen wird, die über Bergson und Deleuze bis Guattari reicht. Affekte werden in dieser Tradition nicht als innerliche Zustände im individuellen Subjekt betrachtet, sondern stets 
,as relations of affecting and being affected between co-evolving bodies in the immanence of the one 'substance' (or 'nature')" (ebd.).

Innerhalb dieser Traditionslinie wird im Anschluss an Spinoza die Unterscheidung zwischen Körper und Geist aufgehoben (Spinoza 2015, Teil III). Blackman trägt in ihrer Definition dem immateriellen Aspekt Rechnung:

,[A]ffect refers to those registers of experience which cannot be easily seen and which might variously be described as non-cognitive, trans-subjective, non-conscious, non-representational, incorporeal and immaterial“" (Blackman 2012: 4).

Affekte bilden demnach eine undefinierte Kraft zwischen Subjekten, deren Persönlichkeiten und Neigungen unaufhörlich in einem wechselseitigen, relationalen Zusammenspiel durch sie verändert werden - wie bei einem unsichtbaren Bindemittel, das die Welt zusammenhält (Massumi 2002: 217). Der Körper nimmt fortwährend Informationen des Umfelds über sein perzeptuelles System auf, so dass der Leib kontinuierlich auf Veränderungen der ,,affective modes“ reagiert, was dem ,meaning-making“, also sinngebenden affektiven Bezugnahmen, gleichkommt (Scheve 2016: 13). Affekte repräsentieren neben dem Aspekt der Zwischenleiblichkeit gleichsam unterschiedliche Erfahrungsmodi, was die Nicht-Intentionalität der Affekte hervorhebt (vgl. Leys 2011). Nicht-Intentionalität weist darauf hin, dass Affekte nicht notwendigerweise einen Objektbezug benötigen, obwohl umgekehrt Objekte Affekte auszulösen in der Lage sind. Der Philosoph Jan Slaby erweitert diese Sicht mit seinem Konzept der genuinen affektiven Intentionalität oder ,,affektiven Grundhaltung“ (2011), das nicht bloß spezifische Objekte berücksichtigt, sondern den gesamten Weltbezug des wahrnehmenden Subjekts erfasst. Dieser Gedanke findet sich besonders in der Vorstellung einer permanenten Hintergrundaffektivität in Form eines ,existenziellen Gefühls“ (Ratcliffe 2008) wieder. Ratcliffe baut diesen existenziellen Modus in seine Theorie (Abschn. 4.2.4) ein, während die bedeutungsstiftende Ebene von Affekten nicht an Wichtigkeit verliert. Dieser Umstand ist, wie Wetherell konstatiert, insofern relevant, als in manchen Ansätzen der Eindruck entsteht, Affektivität sei von sozialer Sinngebung unabhängig (Wetherell 2012). Affekte treten demnach immer im Zusammenhang mit Bedeutungsstiftung auf (Heise 2007; Barrett/Bliss-Moreau 2009). Als „körperliches Resonanzgeschehen“ können Affekte kaum von Gefühlen unterschieden werden. In Anlehnung an das im SFB 1171 entwickelte Begriffsverständnis verstehe ich ein Gefühl als subjektives, leibliches Erleben einer 
spezifischen affektiven Relationalität (Röttger-Rössler 2016: 7). Wenn die affektiven Bezugnahmen auf das Umfeld ein solches Ausmaß erreicht haben, dass das wahrnehmende Subjekt sie bewusst registriert, aber (noch) nicht spezifizieren und als distinkte Emotionen einordnen kann, handelt es sich um ein Gefühl (ebd.). Gefühle werden bewusst wahrgenommen und unterscheiden sich in diesem Punkt von Affekten, die aufgrund der Unbestimmtheit ansonsten recht ähnliche Phänomene darstellen.

Mit dieser Begriffsbestimmung bilden Affekte, Gefühle und Emotionen Gesichtspunkte desselben empirischen Phänomens, wobei mit jeder Perspektive jeweils verschiedene Facetten beleuchtet werden (Röttger-Rössler 2016: 6). Aus diesen Gründen ist ein zu starres Festhalten an den terminologischen Unterscheidungen und Konzeptionen für die vorliegende Studie nicht ratsam. Sie werden als Handwerkszeug begriffen, mit dem die empirisch gewonnenen Daten bearbeitet, beschrieben und analysiert werden sollen, um die Lebenswelten meiner Interviewpartner*innen zu rekonstruieren. Auf der Grundlage des Affektverständnisses werden die Konzepte der „affektiven Relationalität“ sowie des „affektiven Arrangements“ (Slaby 2019; Slaby et al. 2016) als explorative Grundkonzepte angewendet, um die einzigartigen affektiven Facetten von Lebenswelten aus einer sozialphänomenologisch-transpersonalen Perspektive zu beleuchten. Ein affektives Arrangement ist dabei als eine Präzisierung der Idee von affektiver Relationalität zu begreifen. Der Begriff affektive Relationalität bezieht sich auf die Transpersonalität von Affekten. Dies bedeutet vor allem, dass Affekte keine innerlichen ,psychischen“ Zustände, sondern ein komplexes, prozesshaftes, relationales Netzwerk darstellen (ebd.: 74), das zwischen den Körpern der Akteur*innen im Rahmen eines sozial-relationalen Konstitutionsgeschehens besteht (ebd.: 70). Aus diesem affekttheoretischen Blickwinkel werden Affekte in der Lebenswelt der betrachteten Individuen als relationales - und nicht in der Psyche des Subjekts befindliches - System zwischen dem Subjekt, anderen Akteur*innen und den Mechanismen der Institution Asyl verortet, die sich wechselseitig affizieren und voneinander affiziert werden (Lünenborg et al. 2018: 16). Ins Zentrum der Aufmerksamkeit rücken damit spezielle Dynamiken von Affekten in situativen Praktiken. Neben Humankörpern werden mitunter auch andere organische und unorganische Körper einbezogen, etwa technologische oder architektonische Entitäten (Slaby 2016: 3). Die Affekte werden demgemäß zwischen den organischen Körpern der Akteur*innen und zugleich in den einzelnen Körpern effektiv wahrnehmbar (Wetherell 2012: 159). Sie sind daher immer in einem doppelten Sinn wirksam: mental und körperlich (Wiemer 2006: 142). Darauf aufbauend stellen affektive Arrangements materiell-diskursive Gebilde dar, die Affekte derart strukturieren und kanalisieren, dass sie periodisch auftreten 
und durch ihre Intensität die unterschiedlichen Akteur*innen dynamisch miteinander verbinden (Slaby et al. 2016: 3). Sie sind im sozialen Leben allgegenwärtig. Das Konzept der affektiven Arrangements geht zurück auf die Arbeiten von Gilles Deleuze und Felix Guattari (Deleuze/Guattari 1986; 1987), Althusser (1970) sowie Foucault (1980). Althussers Konzept des Apparatus wurde von Foucault aufgegriffen und als Dispositiv theoretisiert (Slaby 2019: 112). Die erwähnten Konzepte weisen auf eine heterogene Gesamtheit von Diskursen, Institutionen, Praktiken und Einrichtungen hin, die Macht etablieren. Foucaults Dispositiv ermöglicht eine relationale, subtile und zugleich polyzentrische Sichtweise auf Machtstrategien, Wissenstypen und Subjektwerdung (siehe Abschn. 4.6) durch die Einbeziehung des Ungesagten und Verdrängten (Foucault 1977b: 392). Mithilfe des Konzepts der affektiven Arrangements lassen sich spezifische soziale lokale Konstellationen untersuchen und aufdecken, die mit intensiven Affekten assoziiert werden (Slaby 2019: 116). Affektive Arrangements fokussieren soziale Verhaltensmuster, die dazu beitragen, affektive Formationen für einen bestimmten Ort und eine bestimmte Zeit zu modellieren (ebd.). Aus diesem Grund eignet sich der Ansatz besonders für die empirische Sozialforschung (ebd.: 115).

Als ein Oberbegriff für alles Empfundene werden auf Farsi die Begriffe

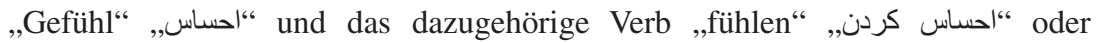
“verwendet. Beide werden weitestgehend synonym gebraucht. In der Muttersprache meiner Interviewpartner*innen wird demnach nicht zwischen Emotion, Affekt und Gefühl unterschieden. Das genannte Begriffspaar auf Farsi (Gefühl und fühlen) wird in den Transkriptionspassagen der Interviews als „Gefühl“ bzw. „fühlen“ übersetzt, da der Begriff auf der semantischen Ebene weitverbreitet ist. Bei der darauf folgenden Analyse werden die Empfindungen aus dem Blickwinkel der verwendeten theoretischen Ansätze und der Daten entsprechend (um-)gedeutet. Dies sollte im Zusammenhang mit der Begriffs- und Konzeptklärung nicht unerwähnt bleiben.

\subsubsection{Emotion}

Obwohl in Bezug auf die Bedeutung des Begriffs Emotion ebenfalls keine Einigkeit in der Wissenschaft herrscht, besteht jedoch weitestgehend Übereinstimmung, dass das Entstehen einer Emotion mit vielen unterschiedlichen Faktoren zusammenhängt. Eindimensionale Ansätze, die sich entweder dem universalistischen oder dem konstruktivistischen Programm verschrieben haben, sind heutzutage in der Emotionsforschung selten. Ihnen liegt ein hierarchisches 
Zwei-Schichten-Denken (vgl. Röttger-Rössler 2004: 15) zugrunde, das einer der beiden theoretischen Richtungen mehr Bedeutung schenkt, indem entweder die körperlich-biologische oder die soziokulturelle Dimension fokussiert wird. Bei den zwei Hauptströmungen handelt es sich einerseits um die biologisch determinierte, universalistische Perspektive (vgl. Izard 1977; Ekman 1970, 1992, 1993), andererseits um die kulturell bedingte, konstruktivistische Sicht (Abu-Lughod 1999; Lutz 1988). Doch das dualistische Denken ist weder pragmatisch noch zielführend, besonders im Rahmen einer empirischen Studie. Ungeachtet dessen, dass es epistemologisch sinnlos ist, einem dieser Ansätze den Vorzug zu geben, wurden bereits beide Sichtweisen als reduktionistisch zurückgewiesen, indem empirische Inkonsistenzen und Unstimmigkeiten nachgewiesen wurden (z. B. Lyon 1994; Leavitt 1996; Reddy 1997; Röttger-Rössler 2002, 2004; Milton 2005; Engelen et al. 2009). Hinzu kommen subjektive Abhängigkeitsverhältnisse während des Forschungsprozesses vor dem Hintergrund unterschiedlicher ideologischer Traditionen, das persönliche Erkenntnisinteresse, die akademischen Voraussetzungen sowie die entsprechend erlernten Erhebungsmethoden. All dies stellt eine Studie von Beginn an in einen erkenntnistheoretischen Zusammenhang, bei dem die Ergebnisse lediglich verschiedene Ordnungen einer Realität oder Erfahrung zum Ausdruck bringen (Shweder et al. 1997: 140), ohne Anspruch auf Deutungshoheit zu erheben.

Der Begriff der Emotion bezeichnet selektiv fokussierte affektive Episoden, die in diskursiv ausgehandelte Rubriken klassifiziert wurden, welche ihrerseits auf bereits bestehenden Wissensbeständen beruhen (Slaby et al. 2016: 77). Emotionen werden konkret als Zusammenspiel von leiblichen Empfindungen und komplexen Konzepten verstanden, die ihrerseits diese Empfindungen heranbilden, verändern und kanalisieren (Röttger-Rössler 2016: 5). Emotionen bilden demnach leibliche Erlebnisdimensionen, die durch soziale und kulturelle Konzeptualisierungen beeinflusst und geformt werden und das wahrnehmende Subjekt in Beziehung zu anderen Akteur*innen, Dingen und Ereignissen setzen (ebd.). Angesichts des dynamischen multidimensionalen Bezugsgeschehens bilden Emotionen ein relationales, prozesshaftes Phänomen. Sie haben demgemäß stets eine kulturelle Konzeptualisierung bzw. Kategorisierung erfahren und unterscheiden sich darin von Affekten (SFB Affective Societies 2016: 3). Angst, Freude, Scham, Zorn, Neid und Stolz sind Beispiele für Emotionen, bei denen sich der affektive Weltbezug facettenreich mittels kulturspezifischer Kategoriensysteme entfaltet (Slaby et al. 2016: 77). Die entsprechenden Erfahrungsdimensionen der affektiven Weltbezüge spiegeln sich in den unterschiedlichen Emotionskategorien wider, die - im Unterschied zu Gefühl und Affekt - immer auf differenzierte normative Systeme verweisen (ebd.). Diese normativen und 
gleichsam epistemischen Ordnungen beurteilen, belohnen und sanktionieren aus affektiven Episoden herangebildete Emotionen (ebd.). Emotionen spiegeln demgemäß bestimmte Weltverhältnisse wider, die durchaus als ambivalent und undurchsichtig erscheinen können (Scheve/Slaby 2019: 43). Das Verhältnis einer Person zu einer gefährlichen Situation, die sie mit Angst erfüllt, definiert beispielsweise ihr charakteristisches Verhältnis zu der betreffenden Gefahr und ist gleichzeitig Ausdruck ihrer Haltung im Hinblick auf persönliche Wünsche und Ziele (ebd.). Emotionen werden als Ergebnis einer aktiven, dynamischen und verkörperlichten Interaktion zwischen Individuen und ihrer jeweiligen Situation betrachtet (ebd.: 43). Im Unterschied zu Stimmungen (moods, sentiments) lassen sich Emotionen daher als „episodisch“ begreifen; sie stellen mithin die Verwirklichung und Konzeptualisierung von Affekten dar (ebd.: 44).

Eine Emotion wird in der vorliegenden Arbeit als ein Multikomponentensystem begriffen, das sich aus fünf grundlegenden Bausteinen konstituiert: der subjektiven Einschätzung (appraisal) (1), der Körperreaktion (2), der Ausdrucksweise (3), der Handlungstendenz (4) und dem Gefühl (5) (siehe Abschn. 4.2.2). Dabei werden die unterschiedlichen Kontexte, etwa der soziale, der kulturelle oder der biologische, als grundsätzlich gleichwertig verstanden, ohne dabei Vollständigkeit in der Untersuchung beanspruchen zu wollen. Dieses Emotionsmodell veranschaulicht den dynamischen, relationalen und prozesshaften Charakter des vielschichtigen, nicht leicht zu fassenden Phänomens Emotion. Es hilft, die Kluft zwischen den zwei gegenläufigen epistemologischen Positionen (vgl. Lutz/ White 1986; Reddy 1997; Röttger-Rössler 2002, 2004; Milton 2005) innerhalb der Emotionsforschung zu reduzieren. Die Veränderung des Blickwinkels von einem ontologischen zu einem systemischen oder relationalen Ansatz beeinflusste maßgeblich die Entwicklung integrativer Emotionsmodelle (Scheidecker 2017: 21). Was nun letztlich eine Emotion ist, kann nach wie vor nicht zweifelsfrei beantwortet werden. Einzelne Emotionen sind keine bewegungslosen Bausteine, die durch genügend Forschung zu einer fixen Einheit zusammengestellt werden könnten. Es steht vielmehr die Frage im Vordergrund, auf welche Art und Weise unterschiedliche Aspekte von Emotionen miteinander agieren und inwiefern soziale, kulturelle oder andere Systeme auf entstehende Emotionen einwirken (Frijda et al. 2004: 465). Das lebendige, dynamische Element verschiedener Emotionsdimensionen ist in der vorliegenden Studie besonders von Interesse. Passend zur methodologischen Verfahrensweise des explorativen Interviews scheint ein multidimensionaler theoretischer Ansatz angemessen zu sein. Zudem ergibt sich durch die Einbeziehung dramaturgischer Elemente aus Katz' Konzept, auf das weiter unten noch einzugehen ist (Abschn. 4.2.3), eine geeignete Perspektive, relevante Ereignisse in Bezug auf Emotionen und emotionale 
Ausdrucksweisen in alltäglichen Situationen umfassend zu beschreiben und gleichzeitig hinreichend flexibel für Veränderungen und Überraschungen zu sein. Diese theoretische Ausrichtung lässt zu, neue empirische Befunde im Sinne einer Bottomup-Analyse in die Untersuchung miteinzubeziehen (vgl. Shweder et al. 2007). Im Unterschied zur Top-down-Herangehensweise erlaubt eine Bottom-up-Analyse die Herausstellung von kulturspezifischen Emotionen aus dem Feld heraus. Dies passt zur gewählten Methodologie dieser Studie. Es ist wichtig, sich an dieser Stelle zu vergegenwärtigen, dass Modelle bzw. theoretische Ansätze keine ideologischen Denktraditionen darstellen, die es zu verteidigen gilt.

\subsubsection{Emotionsmodell: Emotion als Multikomponentensystem}

Das Emotionsmodell, das den Ausführungen dieser Arbeit zugrunde liegt, ist eine Kombination aus den Begriffserläuterungen und den Ausführungen des Berliner Forschungsteams Affective Societies sowie den Arbeiten von Holodynski und Friedlmeier (2006), Shweder et al. (2007), Scheve (2011), Mesquita und Markus (2004) sowie Scheidecker (2017). Es besteht aus fünf Hauptkomponenten:

1) Einschätzung (appraisal)

2) Körperreaktion

3) Ausdruck

4) Handlungstendenz

5) Gefühl

Die synchrone Aktivierung und reziproke Beeinflussung dieser Bausteine resultiert laut dem dynamisch-systemischen Emotionsparadigma in einer einzelnen Emotionsepisode (Holodynski \& Friedlmeier 2006: 27). Besonders wichtig ist bei diesem Vorgang das erste Element, die subjektive Einschätzung. Das Modell berücksichtigt das transpersonale Moment des erlebenden Subjekts insofern, als die Dynamik einer Emotionsepisode unmittelbar von sozialen bzw. kulturellen Faktoren beeinflusst wird.

\section{1) Einschätzung (appraisal)}

Die Annahme der Einschätzung als zentraler Emotionsauslöser ist auf die Arbeiten Magda Arnolds (1960) und Richard Lazarus (1968) zurückzuführen und bezeichnet die einschätzende Bewertung eines Ereignisses oder einer Situation auf der kognitiven Ebene, insbesondere unter Berücksichtigung eigener 
Absichten, Ziele, Wünsche und Überzeugungen (Scherer 1984; Frijda 1986). Emotionsepisoden entstehen je nach bewusster, unbewusster, schemenhafter oder reflexhafter Bewertung bzw. Einschätzung der Bedeutsamkeit des Geschehens. Dennoch sind Appraisalprozesse von reflexhaften Bewertungsprozessen (Scherer 1994) und von bewusst vollzogenen Bewertungen der motivrelevanten Person-Umwelt-Beziehung zu unterscheiden (Holodynski \& Friedlmeier 2006: 42). Bei bewusst vollzogenen Bewertungen wird die bereits abgelaufene emotionale Einschätzung im Nachhinein mithilfe sprachlich gestützten Wissens rekonstruiert (Lazarus 1991: 144). Die Interpretation des Geschehens findet somit auf verschiedenen Bewusstseinsebenen statt und unterliegt nicht der Kontrolle des Subjekts (Engelen et al. 2009: 29). Einschätzungstheorien beziehen grundsätzlich die Dynamiken der Interaktionspartner*innen sowie ihre spezifischen Gedanken und Gefühle mit ein, so dass der Bewertungsprozess ein hohes Maß an Komplexität erreicht (Scheidecker 2017: 23). Appraisaltheorien ermöglichen eine hohe Variabilität emotionaler Kategorien. Jede spezifische Einschätzung resultiert demnach in einer entsprechend spezifischen Emotion (Scherer 2004: 143). Dabei formen soziale und kulturelle Einschätzungsmuster die Bewertung stark mit. Soziokulturelle Einschätzungsmuster sind von kulturspezifischen Ereignis-, Handlungs-, Interaktions- und Beziehungsmustern abhängig (Shweder 2004: 84). Gemeint sind vorherrschende Vorstellungen über Normen, Werte und Kodes (Markus \& Kitayama 1994), die die vorgenommene Einschätzung prägen. Zu denken ist etwa an die unterschiedlichen emotionalen Reaktionen bei der Frage nach Abtreibung, sobald sich der soziale, kulturelle, politische, gesellschaftliche Kontext ändert, vor allem mit Blick auf das enorme Spektrum der Bedeutungszuschreibungen und der damit einhergehenden Emotionen. Einschätzungen offenbaren somit die subjektive Bedeutung des Eingeschätzten und setzen entsprechende Affekte und Emotionen in Gang (Scheve 2012: 133).

\section{2) Körperreaktion}

Körperreaktion meint eine kaum zu kontrollierende physiologische Reaktion des Leibs, nachdem das Subjekt eine Einschätzung vorgenommen hat. Die physiologische Reaktion hat den Sinn, die Körperfunktionen auf die Transformation der Person-Umwelt-Beziehung auszurichten, und umfasst Vorgänge im autonomen Nervensystem sowie gleichermaßen endokrinologische Prozesse (Holodynski \& Friedlmeier 2006: 43). Einige dieser körperlichen Veränderungen sind für das Auge sichtbar (z. B. ein Schweißausbruch) oder lassen sich messen (etwa die Herzschlagfrequenz; vgl. ebd.). Allerdings ist dies nicht immer der Fall, zumal das Paradigma des Messens im vorliegenden Kontext in die Irre führen kann. Die Manifestation von Emotionen in Form von Körperreaktionen mag sich 
weitestgehend auf der physiologischen Ebene ausdrücken und dahingehend auch „messbar“ sein, doch lässt sich ihr Auftreten damit noch nicht nachvollziehen. Es existiert auch ein Gefühlsleben ohne begleitende Körperreaktionen ${ }^{7}$ (ebd.: 54). Das Erscheinen von Körperreaktionen ist zudem keineswegs rein genetisch oder eine unabänderliche Tatsache, mit der man auf die Welt gekommen ist (Scheidecker 2017: 25). Körperreaktionen - als zweiter Baustein des multidimensionalen Emotionsmodells - werden stets durch Einschätzungen in Gang gesetzt, die ja, wie gezeigt, vom soziokulturellen Umfeld abhängig sind.

\section{3) Ausdruck}

Hierbei geht es um all die unterschiedlichen Formen von Verhaltensweisen, die mit Emotionen assoziiert werden, also Mimik, Blickverhalten, Gestik, Körperduktus, Berührung, der Klang der Stimme und das Verhalten im Raum (Holodynski \& Friedlmeier 2006: 53; Collier 1985). Die verschiedenen Ausdrucksmedien können willentlich nachgeahmt werden, jedoch nicht bei unwillkürlichen Tendenzen (Engelen et al. 2009: 38). Ausdrucksweisen, wie sie vorliegend der dritte Baustein des Emotionsmodells sind, bilden in der Regel eine Form der Kommunikation und stellen eine bestimmte Art des Kontaktes mit einzelnen Elementen der Umgebung her (Holodynski \& Friedlmeier 2006: 60). Als Beispiel kann der Ekelausdruck herangezogen werden (Frijda 1986: 13). Er nimmt entweder direkt - zum Beispiel mit dem Ausdruck des Würgens, des Mundöffnens und des Vorstreckens der Zunge - oder indirekt - wenn etwa Kinder ihren Eltern mimisch kommunizieren, die Fütterung zu beenden - Bezug auf die Umwelt (ebd.: 11). Weiterhin von Bedeutung ist die Tatsache, dass Ausdruckszeichen bei Erwachsenen als Komponenten eines eigenständigen mimetischen Zeichensystems aufzufassen sind (Donald 1993; Raeithel 1994). Sie sind immer in einem kommunikativen System gerahmt und lassen sich von rein sprachlichen Zeichensystemen dadurch unterscheiden, dass ausschließlich die motivrelevanten Aspekte der Person-Umwelt-Beziehungen einer Kultur kodifiziert sind (Holodynski \& Friedlmeier 2006: 60). Ausdruckszeichen und ihre Bedeutungsgewebe sind somit von Kultur zu Kultur verschieden. Holodynski und Friedlmeier heben die semiotische Perspektive hervor, indem sie Ausdrucksreaktionen hauptsächlich die Funktion von Zeichen und nicht von instrumentellen Handlungen zuschreiben (ebd.). Röttger-Rössler fügt die symbolische Ebene

${ }^{7}$ Dies zeigt sich zum Beispiel bei Menschen mit Querschnittslähmung (Chwalisz et al. 1988: 820). 
emotionaler Ausdrucksweisen hinzu und bettet diese stärker in den soziokulturellen Rahmen ein (2004: 93 f.). Menschen sind demnach in der Lage, die eigenen Ausdrucksweisen den expliziten und/oder impliziten sozialen Erwartungen und Regeln, was den Ausdruck betrifft, den sogenannten „display rules“, anzupassen (Ekman \& Friesen 1975: 20).

\section{4) Handlungstendenz}

Der vierte Baustein einer Emotion kann als motivierende Aktionsbereitschaft verstanden werden; Emotionen motivieren demnach zu Handlungen (Frijda 2004; Döring 2003). Wie weiter oben bereits erwähnt wurde, spiegeln Emotionen persönliche Wünsche und Ziele in jenen Situationen wider, in denen sie entstehen; sie offenbaren dem sie erlebenden Menschen, was von elementarer Bedeutung ist (Scheve/Slaby 2019: 45). Die spezifische Emotion konstituiert und bewahrt das Verhältnis des Individuums mit derjenigen Sache oder Person, auf die sich die Emotion bezieht (ebd.). Zentral ist, dass Emotionen eine Handlungstendenz realisieren und nicht reflexartig bestimmte „,vorprogrammierte“ Handlungen auslösen. Aus diesem Blickwinkel sind sie selbst eine Form der agency. Dies hat seine Ursache in der Tatsache, dass Situationen und Verhaltensweisen durch Emotionen mitkonstituiert werden (ebd.). Die auf der kognitiven Dimension vorgenommene Einschätzung erfordert eine adäquate Reaktion des Körpers in Form einer Handlung. Die Handlungstendenz oder Handlungsbereitschaft ist von der Handlung als solcher zu unterscheiden. Es geht beim vierten Baustein der Emotion auch um die motorischen Prozesse der Bewältigungshandlung, welche die Person-Umwelt-Beziehung motivdienlich verändern soll (Holodynski \& Friedlmeier 2006: 42). Im Unterschied zu Holodynski und Friedlmeier wird vorliegend gemäß Scherer (2001) zwischen Ausdrucksreaktionen und Handlungstendenzen unterschieden. Die individuelle Handlungsbereitschaft ist mit Blick auf soziale Normen und Wertvorstellungen zu beurteilen.

\section{5) Gefühl}

Ein Gefühl - feeling - wird als subjektives, leibliches Erleben einer spezifischen affektiven Relationalität verstanden (SFB Affective Societies 2016: 6; Röttger-Rössler 2016: 6). Vermehren sich affektive Bezugsrahmen und werden darüber hinaus auch intensiver, bilden sich Gefühle heraus, die vom wahrnehmenden Subjekt allerdings nicht klar als Emotionen klassifiziert werden können (ebd.). Gefühlen fehlt demnach konzeptuell gesprochen das kognitive Moment, um sie in soziokulturelle Erfahrungsdimensionen zu übersetzen, die durch lokale Emotionswörter benannt und sprachlich ausgedrückt werden 
(vgl. Röttger-Rössler \& Markowitsch 2009). Gefühle können nicht objektiv gemessen werden, sondern sind über die Introspektion zu erfassen, was eine Einschränkung objektiver Kriterien wissenschaftlicher Forschung darstellt (Holodynski \& Friedlmeier 2006: 43). In der Kultur- und Sozialanthropologie wurde daher das Gefühl als einzigartiger Zugang zur Emotion konzeptualisiert (vgl. Levy 1984; Rosaldo 1984), vor allem im Bereich des Emotionsvokabulars (vgl. Wierzbicka 1994). Zudem bleibt das Gefühl ein wesentliches Moment der Emotion, nicht zuletzt, weil es sich über die innerliche Dimension hinaus auf emotionalisierende Faktoren in der Außenwelt bezieht. Denken und Fühlen sind dabei keine Gegensatzpaare. Michelle Rosaldos berühmter Ansatz der embodied thoughts (1984) versteht vor dem Hintergrund der ständigen Bezugnahmen auf die externe Welt Gefühle als Gedanken oder als Sprache des Körpers, wobei in ihrem Ansatz die Trennung zwischen kognitiven und affektiven Prozessen nicht mehr relevant zu sein scheint. Der letzte, der fünfte Baustein des Emotionsmodells ermöglicht dem Subjekt, aktiv auf die Emotionsdynamik einzuwirken. Damit kommt Gefühlen eine Überwachungsund Selbstkontrollfunktion innerhalb der Emotionsregulation zu, wodurch sich die Aufmerksamkeit auf den eigentlichen Anlass, der die Emotion in Gang gesetzt hat, richtet (ebd.). Nur durch diesen fortwährenden Selbstregulationsprozess funktioniert das System Emotion korrekt. Die durch das Gefühl in Gang gesetzte Selbstregulation ist erforderlich, weil sowohl emotionsauslösende Handlungskontexte als auch die motivdienlichen Bewältigungshandlungen in den allermeisten Fällen nicht natürlich bzw. angeboren sind, sondern während der Ontogenese erlernt werden (ebd.). Jede Emotionsepisode muss aus diesem Blickwinkel immer wieder alle Bausteine des Systems Emotion neu durchlaufen, die es dabei aufeinander abzustimmen gilt, und im fünften Baustein Gefühl repräsentiert werden (ebd.). Die Steuerung und Kommunikation von Emotionen und die daraus erwachsenen Handlungen werden demgemäß durch einen reflexiven Bewusstseinsvorgang (Damasio 1999: 279) ermöglicht: dem Gefühl (feeling). Das impliziert, dass das Subjekt die Wahrnehmung des Gefühls bewusst erlebt. Im Unterschied zu Affekten, die in ähnlicher Weise unbestimmt und nicht klar kategorisierbar sind, bilden Gefühle bewusste Reflexionsvorgänge ab, die kurzfristige, situativ gebundene Phänomene, aber auch längerfristige, dispositionale Orientierungen sein können, beispielsweise Gefühle der Entfremdung, Fremdheit, Zugehörigkeit usw. (Röttger-Rössler 2016: 6). 


\title{
4.2.3 Emotionen bei Jack Katz
}

In seinem Buch How Emotions Work (1999) untersucht der amerikanische Soziologe Jack Katz Emotionen in Alltagssituationen anhand qualitativer ethnografischer Studien. Katz integriert Handlungen und Gesten des Menschen mithilfe von Videoaufnahmen in seine Emotionsanalysen (1999: 4). Er kommt zu dem Ergebnis, dass Emotionen ,paradoxerweise“ (ebd.: 7) selbst-reflexive Handlungen und Erfahrungen zur selben Zeit sind, wobei ,the self-reflections in emotions is corporeal rather than a matter of discursive reasoning. Through our emotions, we reach back sensually to grasp the tacit, embodied foundations of our selves“ (ebd.). Katz vernachlässigt in seinem Ansatz diskursive Gesichtspunkte zugunsten leiblicher Dimensionen, obwohl auch qualitative Interviews verwendet werden (ebd.: 8). Mit dieser Perspektive lässt sich folglich eine Diskursivierung von Emotionen vermeiden. Die Gesichtspunkte der Prozesshaftigkeit und Kreativität heben die Tatsache hervor, dass Emotionen für Katz immer kunstvoll und kreativ sind (Albrecht 2010: 83). In seiner Abhandlung verschmelzen Subjekt und Objekt in einem transpersonalen Verhältnis, wobei Katz seine zentralen Fragen, mit denen er sich Emotionen widmet, wie folgt formuliert:

\begin{abstract}
„Every day our emotions confront us with an abiding enigma. On the one hand, emotions come to us seemingly beyond our control. We may be possessed by anger, drenched in shame, taken by something we find hysterically funny, or surprised to find ourselves crying from joy. On the other hand, emotions make up a part of our lives that is intimately subjective. [...] Because it postures the individual dialectically, indicating that he is both an object taken and a subjectivity in the process of defining himself, emotional experience raises two questions. If emotions are not just done to but also done by a person, how does he or she create them? [...] How is it that people do not sense that they are creating their emotions?" (Katz 1999: 1)
\end{abstract}

Die dialektische Herangehensweise an das Phänomen Emotion und der Fokus auf den Emotionsausdruck lassen die Frage nach der aktiven, kreativen, das heißt nicht pathologischen Gestaltung von Emotionen in den Vordergrund rücken. Katz analysiert die Emotionen Wut (anger), Lachen (laughing), Scham (shame) und Weinen (crying), indem er über das Interviewmaterial hinaus durch Filmaufnahmen festhält, was Menschen während emotionaler Episoden tun. Emotionen bilden für Jack Katz demzufolge nicht bloß innerpsychische oder rein neuronale Vorgänge. Sie hängen vielmehr, passend zu allen methodologischen sowie theoretischen Vorannahmen und Überlegungen dieser Studie, mit den Beziehungen einzelner Akteur*innen im Umfeld des wahrnehmenden Subjekts zusammen. Gemäß Katz bestehen Emotionen aus drei Bausteinen (Tabelle 4.1 ): 
Tabelle 4.1 Jack Katz'

Emotionsmodell. (Quelle:

Eigene Darstellung)

\begin{tabular}{l|l|l}
\hline Sinnliche Metamorphose & Katz I & $\begin{array}{l}\text { Soziales } \\
\text { Drama }\end{array}$ \\
\cline { 1 - 2 } Soziale Interaktionen & Katz II & \\
\hline Narratives Projekt & Katz III & \\
\hline
\end{tabular}

Diese drei Bausteine werden als gleichzeitig ablaufende dramaturgisch gemeinte Prozesse verstanden, die nicht voneinander getrennt werden können. ${ }^{8}$ Jack Katz zeigt in seiner Analyse, wie Wut und das dazugehörige narrative Projekt synchron aus sinnlicher Metamorphose und Interaktionsprozessen mit anderen Menschen hervorgehen und ein soziales Drama bilden. Das erste Element, die sinnliche Metamorphose, bezieht sich auf die Transformation des physischen Körpers (sensual metamorphosis). Die sinnliche Metamorphose entwickelt sich aus Interpretation, Erwartung und reziprok unterstellten Wahrnehmungen des Menschen (Knoblauch 2015: 11). Der gewählte Begriff „Metamorphose" deutet an, dass die Leiblichkeit und das Handeln des Emotionen erfahrenden und generierenden Individuums für das Verständnis der Verkörperung der jeweiligen Emotion von Bedeutung sind. Man wird dazu angehalten, die sinnliche Verwandlung zu akzeptieren, in der die Körper von Menschen zu Trägern eigener Erfahrungen werden (ebd.: 19). Dabei hängen die Darstellungen und Interpretationen der in Katz' Buch besprochenen Emotionen eng mit der Sinngebung durch die Inszenierung eines sozialen Dramas zusammen. In seinen Ausführungen zur Entstehung der Emotion Wut wird ersichtlich, wie stark die sinnliche Metamorphose des autofahrenden Menschen mit der Sinnhaftigkeit der Identifikation mit dem eigenen Auto zusammenhängt. Die wütende Person wird gewissermaßen selbst zum Auto, das geschnitten wurde, und bildet darauffolgend ein narratives Projekt heran, um sich zu rächen (Katz 1999, Kap. 1). Die mittels der sinnlichen Metamorphose gebildete Identifikation erlaubt es dem Individuum, sich in diesem Fall als Opfer zu betrachten. In der Diskussion um das Weinen (ebd., Kap. 4 und 6) erklärt Katz die sinnliche Metamorphose in ähnlicher Weise. Hier gibt etwa ein Mörder während eines polizeilichen Verhörs durch seine „Metamorphose des Weinens“ nicht bloß zu, die ermordete Person gekannt zu

\footnotetext{
${ }^{8}$ In der Einleitung von How Emotions Work beginnt Katz zwar in seiner Nummerierung mit dem narrativen Projekt und endet mit der sinnlichen Metamorphose (1999: 5-7), doch findet sich in der Anwendung dieses Schemas in den vier Haupt-Essays keine strikte Reihenfolge. Die hier vorgeschlagene Reihenfolge der drei Emotionsdimensionen (Katz I bis III) entnehme ich der Anlage und Deutung der Hauptkapitel seines Buches.
} 
haben, sondern gesteht mit seiner spezifischen körperlichen Wandlung außerdem die begangene Tat (ebd.: 302). Nachdem der Mörder zu Beginn des Verhörs seine Aussagen gemacht hat, führen ihm anschließend Polizeibeamt*innen die entscheidenden Beweisstücke vor. Dadurch wird ihm klar, dass seine bisherigen Äußerungen und Stellungnahmen hinfällig sind (ebd. 307). Die sinnliche Metamorphose des Weinens hilft ihm zunächst, ein Stück weit Kontrolle über die schwierige Situation im Verhörraum zu erlangen (ebd.: 302). In dieser Situation, in der es schwer ist, die ,richtigen Worte“ zu finden, identifiziert sich das Individuum mit dem „Fluss seiner Tränen“, in dem er zu ertrinken scheint, und sendet somit Signale, die im weiteren Verlauf der Verhandlungen zweckdienlich sein werden (ebd.: 303), beispielsweise indem sie das Wohlwollen der Richter*in während des Prozesses gewinnen. Das narrative Projekt (Katz III) baut schließlich auf der Inszenierung der sinnlichen Metamorphose (Katz I) auf. Letztere bildet sich durch die unangenehme Situation im Verhörraum angesichts unnachgiebiger Polizist*innen, die das Spiel ,good cop - bad cop“ spielen (ebd.: 304) (Katz II).

Die sozialen Interaktionen betreffen in Katz' Ansatz die Resonanz der sozialen Interaktionen des Subjekts und sämtliche Prozesse, die zu ihnen gehören. Von Bedeutung sind hier auch Erwartungen und Interpretationen, die in den Interaktionen mitschwingen (Dietz 2012: 31). Hierbei geht es konkret darum, wie das Subjekt sich selbst und andere in seinem Umfeld wahrnimmt und folglich mit ihnen interagiert und was daraus für die eigene Identität folgt. Durch das Weinen inszeniert der Mörder kunstvoll sein narratives Projekt, das darin besteht, im „Whirlpool“ seiner Tränen zu ertrinken, während er nach und nach weitere Verbrechen gesteht (ebd.: 301 f.). Die sinnliche Metamorphose (Katz I) des Mörders bettet folgerichtig seine unmittelbaren Erfahrungen (Katz II) in ein narratives Projekt (Katz III). Mithilfe der Narration kann die Situation so umgewandelt werden, dass sie für ihn Sinn ergibt. Seine Emotion ist seinem Denken nicht antagonistisch gegenübergestellt, sondern erweist sich als selbstreflexive Handlung, die sich körperlich und nicht diskursiv vollzieht. Narrative Projekte integrieren situationsspezifische Erzählungen und Haltungen; sie antworten auf Situationen und transzendieren sie durch Emotionen derart, dass sich sinnvolle Geschichten herauskristallisieren. Die drei Bestandteile seines zirkulär zu verstehenden Modells zielen demnach auf die Inszenierung eines „,sozialen Dramas“ ab. Emotionen sind insofern immer mit einer bestimmten Narration verknüpft (Knoblauch 2015: 10).

Katz legt seinen Untersuchungen eine naturalistisch-soziale Ontologie zugrunde, mit deren Hilfe so viele relevante Dimensionen wie möglich in die Analyse einbezogen werden können (Katz 2002: 255). Ausgehend von der 
naturalistischen Ontologie nimmt er an, dass die drei erwähnten Dimensionen sinnliche Metamorphose, Interaktion und Narration - Emotionen konstituieren. Die Konzepte der einzelnen Dimensionen entspringen dabei unterschiedlichen wissenschaftlichen Traditionen, und zwar dem Symbolischen Interaktionismus (Georg Herbert Mead), der Phänomenologie (Maurice Merlau-Ponty) und der Psychoanalyse (Sigmund Freud). Während die interaktionistische Soziologie sichtbares soziales Handeln zu analysieren sucht, will die Phänomenologie durch strikte egologische Reflexionen generelle Strukturen der subjektiven Orientierung von Lebenswelten aufdecken. Mithilfe des Freud'schen Ansatzes der Psychoanalyse sollen schließlich unbewusste Mechanismen und Strukturen des Subjekts nachvollzogen werden (Dietz 2012: 37). In der Integration der unterschiedlichen theoretischen Ansätze bleiben zwar viele Fragen unbeantwortet, doch geht es Katz im Grunde um die Frage „What is it that, being itself invisible, produces all that is visible?" (Katz 1999: 309). Während die Psychologie den Körper als Behältnis für Triebe und Bedürfnisse sieht, tendiert die Soziologie dazu, sich auf die Repräsentationen des Körpers im Diskurs zu beschränken (Dietz 2012: 37). Indem aber gefragt wird, wie genau eine Emotion erlebt wird, rücken Wechselwirkungen von Körper und Umwelt in den Vordergrund (ebd.: 334). Die Betonung der situationstranszendierenden Facette einer Emotion, insbesondere deren Prozesshaftigkeit, begünstigt in diesem Ansatz eine multidimensionale Untersuchung: „Emotions are ways of turning back on the self, ways of reflexively amplifying and giving added resonance to the transcendent meaning of situated action“ (ebd.: 332). Mithilfe des sozialen Dramas ermöglicht das Subjekt die Generierung eines spezifischen moralischen Bedeutungszusammenhangs. Dieser wird als allgemeingültig definiert, um die Rechtfertigung zu liefern, dieses oder jenes bestimmte soziale Drama aufzuführen. Letzteres antwortet mitsamt der spezifischen Narration des Subjekts in dieser Perspektive auf herausfordernde Situationen und transzendiert sie (ebd.). Maßgeblich ist, dass die jeweilige Situation, in der die Emotion erlebt wird, mit einem Sinn verknüpft wird. Die Emotion kann erst dann transzendiert werden, wenn ihr ein ganz spezifischer Sinn verliehen wird, der sozial sichtbar ist (Albrecht 2010: 90). Emotionen besitzen demzufolge die Macht, Situationen zu transformieren, indem sie ihre Bedeutung verändern. Sie stellen, anders gesagt, eine aktive Leistung zur Konstruktion einer neuen Bedeutung einer Situation dar (Knoblauch 2015: 11). Diese Auffassung eignet sich für die vorliegende Analyse, weil der Kontext der Studie - Asyl und Flucht - im Regelfall mit negativ besetzten Emotionen wie Scham, Angst, Verzweiflung und Trauer assoziiert wird, die das Subjekt in irgendeiner Form pathologisieren und stigmatisieren. Die drei Komponenten dieses Emotionsmodells implizieren zudem unterschiedliche Denkschulen, wie die Psychoanalyse 
oder den symbolischen Interaktionismus, was für die Ausleuchtung neuartiger, im sozialen Leben weniger offensichtlicher Gesichtspunkte hilfreich ist. Die breite theoretische Ausrichtung ist bei der Interpretation der jeweiligen Elemente des Katz'schen Emotionsverständnisses hilfreich. Ein weiterer Vorteil der Einbeziehung desselben in die vorliegende Ethnografie besteht in dem Blick auf emotionale Ausdrucksweisen des Menschen durch literarisch-dichte Ausarbeitungen der erlebten Situationen. Somit wird dem Umstand Rechnung getragen, dass Emotionen situativ und interaktiv untersucht werden.

\subsubsection{Existenzielle Gefühle nach Matthew Ratcliffe}

Die in der Phänomenologie behandelten dispositionalen Gefühlslagen, die gewöhnlich als Befindlichkeit, Stimmung oder Atmosphäre bezeichnet werden, finden in dem neuen Forschungsansatz der existenziellen Gefühle Ausdruck. Der britische Philosoph Matthew Ratcliffe (1973*) skizziert in seinem Buch Feelings of Being (2008) eine phänomenologische Theorie über existenzielle, für den menschlichen Welt- und Selbstbezug zentrale Hintergrundgefühle. Die sogenannten ,existenziellen Gefühle“ (Ratcliffe 2005) entsprechen permanent anwesenden „Hintergrundgefühlen“ (Ratcliffe 2008). Sie verändern das qualitative Erleben so, dass spezifische Bezugnahmen auf innerweltliche Begebenheiten affektiv vorgeprägt werden (Slaby 2018: 2). Existenzielle Gefühle beziehen sich auf die Welt des wahrnehmenden Subjekts als solche, nicht auf einzelne Gesichtspunkte seines Lebens; sie strukturieren das gesamte Leben. Ausgangspunkt und Inspiration seiner Überlegungen ist Martin Heideggers Reflexion über „Befindlichkeit“ in seinem Hauptwerk Sein und Zeit von 1927. Gemäß Ratcliffe sind existenzielle Gefühle weder bloße subjektive Befindlichkeiten noch auf Ereignisse in der Welt bezogene, intentionale Gefühle oder Emotionen. Sie sind auch nicht als eine Ebene der „Erfahrung“ zu sehen. Existenzielle Gefühle sind diesen Bezugssystemen vorgeordnet. In ihnen sind Erfahrung und Verhalten sowie Erfahrungen von Selbst und Welt noch ungespalten (Slaby 2012: 80). Ratcliffe beschreibt dies in seinen eigenen Worten wie folgt:

„The world as a whole can sometimes appear unfamiliar, unreal, distant or close. It can be something that one feels apart from or at one with. One can feel in control of one's overall situation or overwhelmed by it. One can feel like a participant in the world or like a detached, estranged observer, staring at objects that do not feel quite 'there'. Such relationships structure all experiences. Whenever one has a specifically 
focused experience of oneself, another person or an inanimate object being a certain way, the experience has, as a background, a more general sense of one's relationship with the world. This relationship does not simply consist in an experience of being an entity that occupies a spatial and temporal location, alongside a host of other entities. Ways of finding oneself in a world are spaces of possibility, which determine the various ways in which things can be experienced. For example, if one's sense of the world is tainted by a 'feeling of unreality', this will affect how all objects of perception appear; they are distant, removed, not quite 'there" " (Ratcliffe 2008: $37 \mathrm{f}$.).

Existenzielle Gefühle verleihen Menschen ein implizites Verständnis von Möglichkeiten (Ratcliffe 2008: 68). Gemeint sind Handlungs- und Erfahrungsmöglichkeiten des Subjekts, die präreflexiv erschlossen werden und dessen Wirklichkeitssinn modellieren (Slaby 2012: 79). Existenzielle Gefühle bilden die affektive Grundhaltung und Rahmung menschlicher Erfahrung. Daraus wird ihre Nähe zur Handlungsfähigkeit, und zwar mit einem implizit mitlaufenden aktivistischen Sinn für die eigenen Fähigkeiten, Möglichkeiten und Tendenzen, ersichtlich (Slaby 2011: 29). Nach Ratcliffe eröffnen existenzielle Gefühle ein Spektrum von Handlungsmöglichkeiten (Ratcliffe 2008: 69). Im Ergebnis rücken ganz bestimmte Verhaltensweisen und entsprechend Widerfahrnisse in den Vordergrund des empfindenden Menschen, während andere, entgegengesetzte Verhaltensweisen und Widerfahrnisse ausgeschlossen werden (ebd.: 70). Existenzielle Gefühle determinieren und formen somit präintentional die wesentlichen Erfahrungsmöglichkeiten im Leben eines Menschen (Ratcliffe 2015: 35). Bestimmte Erfahrungsmodi können Betroffene infolgedessen gar nicht mehr erleben oder fühlen. Angesichts der Neigung innerhalb der Emotionsforschung, bestimmte soziokulturell konzeptualisierte Gefühle - also Emotionen - im Zusammenhang mit dem Möglichkeitsbezug zu favorisieren, hebt Ratcliffe hervor, dass es sich bei existenziellen Gefühlen gerade nicht um anerkannte Emotionen wie Freude, Neid, Scham oder Angst (siehe weiter oben) handeln muss (ebd.). Die existenzielle Struktur des Erlebens, die den jeweiligen ,Weltzugriff“ (Slaby 2011: 33) konstituiert, kann auch durch ein Zusammenspiel verschiedener sich wiederholender Gefühle und Affekte geformt werden, die kaum bekannt sind. ${ }^{9}$

\footnotetext{
${ }^{9}$ In der ersten Fallstudie dieser Arbeit wird etwa die badbakhti als ein solches affektives Grundgerüst verstanden, das die existenziellen Gefühle der empfindenden Person ins Leben ruft (siehe Kap. 6). Hier fühlt sie sich unsicher, ängstlich, minderwertig, bemitleidenswert, kläglich, elend, würdelos und hoffnungslos. Die badbakhti wird in Anlehnung an Ratcliffe daher als ein paradigmatisches existenzielles Gefühl innerhalb der Institution Asyl konzeptualisiert, die den Wirklichkeits- und vor allem Möglichkeitssinn betroffener Asylbewerber*innen konstituiert.
} 
Ratcliffe verbindet zudem eine Reihe psychischer und psychiatrischer Krankheiten, zum Beispiel Depression, Schizophrenie, Wahnvorstellungen usw., mit existenziellen Gefühlen. Das konventionelle psychologische Verständnis von Erfahrung und Kognition wird in Ratcliffes Überlegungen durch die Idee existenzieller Gefühle vervollständigt. Dies ist deshalb von Belang, weil es im Kontext psychisch kranker Personen um grundlegend ,andere Wirklichkeiten" (Ratcliffe 2008: Kap.7) geht. Das Selbst fühlt, dass es sich in einer anderen Welt befindet und nicht bloß andere Gefühlszustände ,hat“, und doch kann es diese Information nicht auf sich selbst beziehen. Denn es ist die Welt und all die individuellen Bezüge zu dieser Welt - mithin die gesamte Existenz -, die von Grund auf verändert sind. Körperlich empfundene Gefühle, „bodily feelings“, sind in diesem Ansatz untrennbar von grundsätzlichen Welterfahrungen, „,world-experiences“ (ebd.: 1), und von „,ways of finding oneself in a world" (ebd.: 2). Die Handlungsnähe existenzieller Gefühle wird indes als „sense of possibilities“ (ebd.: 121) beschrieben. Kognitivistische und empfindungstheoretische Emotionstheorien rücken aus dieser Blickrichtung näher zusammen, womit Ratcliffe an eine Reihe von Positionen anknüpft, die die Verschränkung von Gefühl und Kognition hervorheben (Goldie 2000; Helm 2001; Roberts 2003; Döring 2007; Slaby 2008). Systematisch zeigt er die Nutzlosigkeit einer Perspektive, die Emotionen ausschließlich als welterschließende Zustände (Kognition) oder als gespürte Empfindungen (Gefühl) begreift. Emotionen sind für Ratcliffe schlichtweg beides. Entsprechend verweist die Kategorie „existenzielle Gefühle“ auf weitaus mehr als auf das episodische Aufflackern von Empfindungen. Existenzielle Gefühle sind umfassender und prägen den Charakter nachhaltig. Die Hintergrundstrukturen der Affektivität werden von Ratcliffe ausführlich ausgearbeitet und anschließend im Kontext des Tastsinns diskutiert. Hier verdeutlicht er etwa, wie Gefühle leibliche Empfindungsdimensionen sind und zur selben Zeit außerhalb des Körpers wahrgenommen werden (Slaby 2012: 84). Der Leib wird durch die Wahl des Tastsinns in der Analyse - denn hier bleibt die Grenzlinie zwischen Erkennendem und Erkanntem unschärfer als beim Einsatz anderer Sinne - zum Medium des affektiven Weltbezugs (Ratcliffe 2008: Kap. 3). Die haptische Wahrnehmung verleiht Dingen in der unmittelbaren Umgebung zudem besonderes Gewicht und zeigt auf, wie sehr Gefühle in der leiblich-haptischen Ebene zu lokalisieren sind (Slaby 2012: 84). Damit knüpft Ratcliffe an eine Tradition des interpersonalen Verstehens im Anschluss an Husserl und Schütz an. Slaby erwähnt in seiner Analyse von Ratcliffes Ansatz die philosophische Distanz zur geistesgeschichtlich tief verankerten Subjekt-Objekt-Trennung, denn angesichts der recht weitgefassten Sicht Ratcliffes können Gefühle weder dem individuellen Subjekt noch der objektiven 
Welt zugeschrieben werden (Slaby 2012: 80). Der phänomenologische Ansatz versteht die Welt als immer schon affektiv erschlossen und entsprechend der Gefühle des Subjekts eingefärbt, während das Subjekt gar nicht ohne affektive Weltbezüge existieren kann (ebd.). Subjektivität wird immer durch existenzielle Gefühle bedingt. Der Ansatz der existenziellen Gefühle ist somit soziologisch an weltkonstruierende praktische Orientierungen im Zuge der transpersonalen Wirklichkeitskonstruktion und Lebensweltanalyse anschlussfähig.

In Ratcliffes Ansatz werden neben Stärken jedoch auch Schwächen sichtbar. Die deskriptive Ebene steht zu sehr im Mittelpunkt, so dass sein Ansatz nicht systematisch zu einer Theorie ausgearbeitet werden kann (ebd.: 87). Einerseits ist Ratcliffes Einsicht hilfreich, um den affektiven Weltbezug der Lebenswelt eines Subjekts zu rahmen: Existenzielle Gefühle sind ein Element einer weltkonstituierenden praktischen Orientierung und somit ein wesentliches Moment in der sozialen Wirklichkeitskonstruktion des Subjekts (ebd.: 86). Das latente Gefühl der Verletzbarkeit des gefangenen Menschen und der Gefahr durch Angriffe auf das Selbst oder das Gefühl, unaufhörlich den Blicken anderer Menschen ausgesetzt zu sein, beim Empfinden von Scham innerhalb der Institution Asyl können beispielsweise sehr gut mit diesem Konzept beschrieben und verstanden werden. Andererseits bleibt die Idee einer Hintergrundaffektivität im Erleben des Menschen ohne differenzierte Systematisierung der verschiedenen Ebenen zu ungenau, um tatsächlich den Anspruch einer theoretischen Grundlage erfüllen zu können.

\subsection{Der Postkolonialismus}

Dieses Unterkapitel skizziert grundlegende Aspekte des Postkolonialismus, die für das Verständnis zentraler Ideen, Denkfiguren und Schlussfolgerungen dieser Studie ausschlaggebend sind. Die Reflexion des allgemeinen Verständnisses über die Auslegung übergeordneter historisch-politischer Konstellationen ist auch deshalb von Bedeutung, weil es gilt, sich von akademischen Positionen zu distanzieren, die den Postkolonialismus auf eine Frage von „Tischmanieren“ (Spivak 1990: 148) reduzieren.

Der Begriff Postkolonialismus bezeichnete eine Reihe kulturkritischer Arbeiten, die im Nachgang der politischen Unabhängigkeit ehemaliger Kolonien zunächst in den 1940er und 1950er Jahren durch die Kolonialismuskritik und später in den 1970er Jahren durch die Imperialismustheorie beeinflusst wurden (Bienfait 2006: 89). Der dezidiert antikolonialistische Kampf gegen europäische Kolonialherren, gegen Unterdrückung, Ausbeutung und Sklaverei bildet seit 
Beginn der postkolonialen Kritik (vgl. Young 2001) den primären Movens für die Arbeiten postkolonialer Theoretiker*innen (Struve 2013: 13). Die Postcolonial Studies schließen an die Vorarbeiten der Cultural Studies an und fokussieren seit den 1980er Jahren die Themenbereiche Rassismus, Migration und Diaspora (ebd.). Postkolonialismus soll jedoch nicht als eine singuläre Episode verstanden werden, die erst „nach“ dem Kolonialismus eingetreten sei, sondern vielmehr als ein Spektrum von Widerstandsformen gegen die koloniale Herrschaft mitsamt ihren Implikationen und Folgen (Castro Varela/Dhawan 2015: 16). In diesem Zuge wird die Sichtweise vertreten, die moderne Geschichte als ein Ensemble von komplexen Verflechtungen zu begreifen. Aus diesem Blickwinkel ist es nicht weiter möglich, die Geschichte des Westens ohne die Geschichte der kolonisierten Länder und Gesellschaften zu schreiben, was in gleichem Maße aber auch umgekehrt gilt (Conrad/Randeria 2002: 17). Der Postkolonialismus widmet sich daher den Paradoxien und Schwierigkeiten des Dekolonisierungsprozesses, ohne dessen Vielschichtigkeit und Uneindeutigkeit in Abrede zu stellen. Ein uniformes Verständnis des Postkolonialismus ist auch aus diesem Grund wenig sinnvoll (Castro Varela/Dhawan 2015: 16).

Dennoch zielen postkoloniale Überlegungen in ihren Grundannahmen auf die Kritik eurozentrischer und neokolonialer Bestrebungen und Strategien, die mit entsprechenden Haltungen und Vorstellungen einhergehen (ebd.). Hier rücken vor allem Problematisierungen dominierender Diskurse zu Sprache, Ethnie und sozialer Klasse ins Zentrum des Interesses postkolonialer Theoretiker*innen (ebd.: 17). Postkoloniales Denken reflektiert die Kolonialgeschichte als Geschichte der Unterwerfung durch Fremdherrschaft, wobei nicht bloß ökonomische Interessen den Mittelpunkt der Aufmerksamkeit bilden, sondern vielmehr menschenverachtende und rassistische Denk-, Verhaltens- und Herrschaftsmuster (Stiegler 2015: 110). Postkolonialität bezeichnet keine konkrete historische Epoche, sondern ein theoretisches Paradigma oder einen Denkmodus, dessen Ausgangspunkt die europäische Expansionspolitik darstellt (Kaltmeier 2012: 203). Es wird keineswegs angenommen, die Kolonialgeschichte und die damit verbundenen Haltungen seien vollständig abgeschlossen, wenngleich sich die ehemaligen Kolonien nach dem Zweiten Weltkrieg schrittweise in blutigen Kämpfen von ihren „Kolonialherren“ abkoppeln konnten. Die erwähnte Unterwerfung von Minderheiten wird gegenwärtig durch neuartige Mechanismen und Methoden fortgesetzt und ist in westlichen Diskursen omnipräsent. In diesem Kontext seien vorherrschende Gesinnungen und Repräsentationsregime erwähnt, die zahlreiche Formen von Diskriminierungen an Menschen, deren religiöse, ethnische, nationale und geschlechtliche Zugehörigkeit nicht der westlichen Norm entspricht, ermöglichen und produzieren (Hall 2012, Kap. 1). 
Der Postkolonialismus untersucht demnach nicht bloß Herrschaftsbeziehungen zwischen (ehemaligen) „Kolonisatoren“ und „Kolonisierten“; er fokussiert vielmehr, wie sich diese in Form rassistischer und fremdenfeindlicher Gesinnungen auf globaler, nationaler und lokaler Ebene fortsetzen (Winkel 2019: 294). Die ständige Angst vor „dem Anderen“ - ob Chinese, Sinti und Roma, Araber, Afrikaner oder Jude - führt in weiten Teilen westlicher Gesellschaften zu einem spezifischen Bild von den jeweiligen Minderheiten und schafft so aktiv Sinn- und Bedeutungshorizonte (Winter 2012: 132). Angesichts des neuen entfremdeten Selbstbildes bestätigen die derart diskriminierten Minderheiten die Zuschreibungen durch die ,weißen Herrscher“ (vgl. Fanon 1980; Taylor 1992). Daraus entstehende Dynamiken und Verflechtungen in der globalisierten Welt des 21. Jahrhunderts drücken sich mitunter in Macht- und Ausbeutungsverhältnissen aus, die kritisch denkende Menschen nicht zu Unrecht sagen lassen, der Kolonialismus sei niemals abgeschlossen worden. Diese Dynamiken beeinflussen Formen der Staatsführung, die bestimmte Vorstellungen von Identität und Kultur favorisieren und zugleich konträre Ideen und Konzepte diskreditieren, um „den eigenen" Status quo aufrechtzuerhalten. Unter diesem Gesichtspunkt zielt der Postkolonialismus, ähnlich wie der Dekonstruktivismus (Derrida), die Diskursanalyse (Foucault) oder die Kritische Theorie (Adorno und Horkheimer), auf die radikale Kritik und Dekonstruktion von Modellen modernen binären Denkens. Zu diesen einflussreichen erkenntnis- und politikleitenden Binarismen gehören etwa Weiß und Schwarz, Okzident und Orient, Mann und Frau und so weiter (Stiegler 2015: 110), die für rassistische, sexistische und klassistische Diskurse ausschlaggebend sind (Hall 2012: 22). Angesichts der Komplexität (neo-)kolonialer Verwicklungen, die der Postkolonialismus beschreibt und untersucht, bleibt er politisch stets ambivalent und lässt sich nicht ohne Weiteres kategorisieren. Die vorliegende Studie knüpft in ihrem Verständnis des Postkolonialismus an Stuart Hall an, der die zentrale Bedeutung dieses theoretischen Paradigmas mit Erfahrungen der Diaspora, der Dezentrierung und der Globalität verknüpft und unter anderem eine Veränderung der Erzählperspektive fordert (Hall 1997: 231). Der kritische Blick auf subalterne Erfahrungen und Perspektiven des „Andersseins“ spielt dabei eine zentrale Rolle: „Thinking about my own sense of identity, I realize that it has always depended on the fact of being a migrant, on the difference from the rest of you $[\ldots]$, the colonized subject is $[\ldots]$ always other" (Hall 1987: 114-115). Die Aufgabe postkolonialer Theoretiker*innen bestehe dementsprechend in einer umfassenden Kritik am eurozentrischen Blick auf die Welt und an der daraus resultierenden Konstruktion (westlicher) Identitäten und (nicht westlicher) Alteritäten (Purtschert 2012: 343-344). 
Ausgehend von dieser Agenda soll postkoloniales Denken in dieser Studie auf den Bereich Asyl und Flucht in Deutschland angewendet werden. Die Verknüpfung der empirischen Ergebnisse mit postkolonialen Ansätzen und die Auseinandersetzung mit (post-)kolonialen Diskursen mag zunächst nicht ohne Weiteres einleuchten. So lässt sich dagegen argumentieren, dass Deutschland nur eine nachgeordnete Kolonialgeschichte habe (Bronfen et al. 1997), und bezogen auf die vorliegende Untersuchung könnte darauf verwiesen werden, Deutschland sei niemals Kolonialmacht in Afghanistan gewesen. Doch neben diesen berechtigten Einwänden gilt es, einen anderen Gesichtspunkt, der für den Diskurs zu Fluchtmigrierenden in der Bundesrepublik nicht unerheblich ist, hervorzuheben. Neben der Kolonialgeschichte Deutschlands, die erst 1884/85 mit der Kolonialisierung Togos, Kameruns, „Deutsch-Südwestafrikas“, „Deutsch-Ostafrikas“ und „Deutsch-Neuguineas“ begann ${ }^{10}$ und mit der Aberkennung der eroberten Gebiete durch den Versailler Vertrag 1919 endete, haben sich neue Formen des Kolonialismus ergeben (Ha 2003: 63). Diese manifestieren sich etwa in der diskriminatorischen Arbeitsmarkt- und Migrationspolitik als „Inversion kolonialer Expansionsformen“ (ebd.: 64), die als ,innere Kolonialisierung“ (ebd.: 63) der Bundesrepublik gedeutet wird. Aus einer ehemals nachfrageorientierten Arbeitsmarkt- und Migrationspolitik entwickelt sich demgemäß immer mehr eine systematische Selektion von Humankapital $^{11}$. Die selektive Haltung Deutschlands bringt einen Mobilitätsgewinn für wenige hochqualifizierte Menschen und wirkt zugleich wie eine Barriere für alle anderen Gruppen (Hoesch 2018: 21). Im Zuge der Globalisierung öffnen sich die deutschen Grenzen somit für Kapital und Güter, doch nicht für Menschen außerhalb der privilegierten OECD-Staaten (ebd.: 5), die erwähnten „Hochqualifizierten"12 (Humankapital) ausgenommen. Diese Form der selektiven

\footnotetext{
${ }^{10}$ Hinzu kamen noch einige Inseln im Pazifik (vgl. Grosse 1997).

${ }^{11}$ Deutschland verfolgte über Dekaden einen nachfrageorientierten Ansatz in der Migrationspolitik; stark vereinfacht bedeutet dies, dass jede Person mit einem Arbeitsvertrag nach Deutschland einwandern durfte. Seit 2012 änderte sich dies mit Artikel 18c des Zuwanderungsgesetzes, in dem eine Aufenthaltsgenehmigung eigens für die Jobsuche vorgesehen ist. „Hochqualifizierte“ (siehe nächste Fußnote) dürfen sich in der Konsequenz auch ohne Arbeitsvertrag in Deutschland aufhalten (Hoesch 2018: 165 f.).

${ }^{12} \mathrm{Nach} \S 19$ AufenthG sind „Hochqualifizierte“ vor allem „Wissenschaftler mit besonderen fachlichen Kenntnissen oder Lehrpersonen in herausgehobener Funktion oder wissenschaftliche Mitarbeiter in herausgehobener Funktion“ (BAMF 2019). Dieser Personengruppe kann bei einer Einreise nach Deutschland umgehend eine Niederlassungserlaubnis erteilt werden, die sie zum dauerhaften Aufenthalt in Deutschland berechtigt (ebd.).
} 
Arbeitsmarkt- und Migrationspolitik resultiert im Anstieg illegaler Migration und führt insbesondere zu einer Vermischung von Asyl- und Wirtschaftsmigration.

An dieser Stelle wird der Kern des humanitär gedachten Asylgesetzes unterminiert. Deutlich wird diese Tatsache unter anderem am Status zahlloser abgelehnter Asylbewerber*innen, deren Bleiberecht an eine Schulausbildung oder vergleichbare Ausbildung gekoppelt ist, etwa durch den § 25a AufenthG. Der Deutsche Bundestag verabschiedete am 3. Juni 2016 das sogenannte Integrationsgesetz der Großen Koalition, das die wirtschaftliche Seite der Asylpolitik explizit erwähnt: „Der deutsche Arbeitsmarkt benötigt eine Vielzahl von Fachkräften. Dieser Bedarf kann auch durch die nach Deutschland kommenden schutzsuchenden Menschen teilweise abgedeckt werden“ (BT-Drs. 18/8829 (2016): 1). Wenn Asylsuchende demnach die deutsche Sprache lernen und für ihren Lebensunterhalt selbst aufkommen, dürfen sie an der Gesellschaft teilhaben. Traumatisierte und psychisch beeinträchtigte Fluchtmigrierende, die nicht ohne Weiteres in der Lage sind, eine neue Sprache zu lernen und zu arbeiten, werden durch das „Integrationsgesetz“ aussortiert. Es sieht ferner vor, die monatlichen Sozialleistungen von Asylsuchenden, die nicht regelmäßig an den Sprachund Integrationskursen teilnehmen, zu kürzen bzw. zu streichen, ohne etwaige besondere Umstände - etwa psychische, körperliche und/oder kognitive Beeinträchtigungen - zu berücksichtigen. Die zielgerichtete Vermischung des Asylrechts mit arbeitsmarktpolitischen Bedürfnissen und die mit ihr verbundene Selektion und Hierarchisierung von Menschen wird in dieser Arbeit als Facette der erwähnten ,inneren Kolonialisierung“ verstanden. ${ }^{13}$ Die Rekrutierung ,nützlicher" Menschen bildet eine Dimension des uneingelösten Versprechens des Schutzes und der Gleichheit vor dem Gesetz, das die humanitäre Essenz von Asylgesetzen erodieren lässt, worauf im weiteren Verlauf der Arbeit zurückzukommen sein wird.

In der Konsequenz wird eine Form der Macht herangebildet, innerhalb derer Fluchtmigrierenden aus einer überlegenen Position heraus subalterne Rollen zugeteilt werden. Der postkoloniale Untersuchungsansatz stellt sich in diesem Licht als eine deskriptive und nicht als evaluative Herangehensweise dar (Hall 1997: 226). In der Folge lassen sich zwei Dimensionen von Postkolonialismus erkennen: erstens die historische Dimension mit einer zeitlichen Beziehung zwischen einer Kolonie und einem postkolonialen Staat und zweitens eine kritische oder epistemologische Dimension, in der die postkoloniale Theorie

\footnotetext{
${ }^{13}$ Zur Vermischung wirtschaftlicher und humanitärer Interessen Deutschlands in Afghanistan siehe Abschn. 3.1.1.
} 
zum Tragen kommt, um Diskurse über den Umgang mit dem kulturellen Anderen in einem machtasymmetrischen Rahmen zu untersuchen (ebd.: 237). Zentrale Themen des kritischen Postkolonialismus sind daher Alterität und Identität, Diaspora, Rassismus, Klassismus, Sexismus, Hybridität und Globalisierung (Struve 2012: 90). Der kritische Postkolonialismus definiert sich über theoretische Zugänge zu jedwedem Gegenstand, der solche Machtasymmetrien fokussiert (Dietze 2005: 304). Die Fluchtmigration, mit der Europa in der Gegenwart konfrontiert ist und in Zukunft weiterhin konfrontiert sein wird, erinnert an jene Machtasymmetrien, die im Kontext globaler Ungleichheiten eingebettet sind. Fluchtmigration ruft ins Gedächtnis, dass die Entkolonialisierung längst nicht abgeschlossen ist, und sie lässt diese Erinnerung in westlichen Metropolen lebendig werden (Bhabha 2012: 55). Geflüchtete, auch asylrechtlich anerkannte Asylsuchende, sind in den Ankunftsstaaten ihrer Flucht ethnischer und rassistischer Diskriminierung ausgesetzt und werden von wesentlichen Bereichen der Gesellschaft ausgeschlossen. Die in der vorliegenden Arbeit betrachteten Machtasymmetrien und Abhängigkeiten durch die Strukturen der Institution Asyl knüpfen an die skizzierten Aspekte des kritischen Postkolonialismus an, insbesondere hinsichtlich der Unterwerfung von Minderheiten kulturell differenter Menschen und des damit einhergehenden Diskurses über Geflüchtete in Deutschland.

\subsection{Gayatri Chakravorty Spivak: Die Sprachlosigkeit der "Subalternen"}

Die Literaturwissenschaftlerin und feministisch-postkoloniale Theoretikerin Gayatri Chakravorty Spivak, 1942 in Kalkutta geboren, absolvierte zunächst mit 17 Jahren am Presidency College ${ }^{14}$ ihren Bachelor in Englischer Literatur mit Auszeichnung und daraufhin ihren Master in Anglistik an der Cornell University in den USA (Castro Varela/Dhawan 2015: 151). Als erste Frau erhielt sie 1964 ein volles Stipendium der Telluride Association und promovierte 1974 bei Paul de Man (1919-1983) in Vergleichender Literaturwissenschaft mit einer Abhandlung über das Werk des irischen Poeten William Butler Yeats (1865-1939). Mit ihrer Übersetzung des epochemachenden Werks De la Grammatologie (1967) von Jacques Derrida (1930-2004) in die englische Sprache erlangte sie, gleichsam

\footnotetext{
${ }^{14}$ Heute Presidency University of Kolkata.
} 
mit der Rezeption der Derrida'schen Dekonstruktion, weltweite Anerkennung (ebd.: 152). Seit 2007 besetzt Spivak den höchsten akademischen Rang einer USamerikanischen Hochschule als University Professor an der Columbia University (Dhawan 2016: 173). Mit ihrem einflussreichen Essay „Can the subaltern speak“ (1988) rückt sie Subalterne - begriffen als ein Spektrum innergesellschaftlich marginalisierter und gleichzeitig heterogener Subjektpositionen - in den Vordergrund ihrer Untersuchungen.

Spivaks Konzept der Subalternität wurzelt in den Werken des italienischen Schriftstellers Antonio Gramsci (1891-1937), der in seinen Gefängnisheften (1929-1935) mit dem Begriff „Subalterne“ diejenigen bezeichnet, die keiner hegemonialen Klasse angehören. Mit seinem Ansatz ergänzt der Mitbegründer der Kommunistischen Partei Italiens die orthodoxe marxistische Perspektive um sein profundes Verständnis von Herrschaft und Unterordnung (Dhawan 2016: 172). Die Benachteiligung subalterner Gruppen wird in Gramscis Perspektive über die materielle bzw. ökonomische Exklusion hinaus durch eine Vielzahl von Ausschließungen herbeigeführt (Bieling 2015: 464). Hierzu zählen kulturelle, soziale, wirtschaftliche, politische und alltägliche Gesichtspunkte, mit deren Hilfe Gramsci die Totalität eines dynamischen Herrschaftsgefüges umfassender nachzuzeichnen imstande ist (Jacobitz 1991: 10). Subalterne Gruppen sind nur im Verhältnis zur dominanten Mehrheitsgruppe nachvollziehbar. Letztere unterscheidet sich als geschlossene Einheit von den zahllosen fragmentierten Gruppen Subalterner durch Autonomie, Zugang zu den Schlüsselressourcen Wissen und Bildung sowie politische Organisation (Dhawan 2016: 173). Nicht hegemoniale Gruppen - Subalterne - bilden einen Gegenbegriff zur Hegemonie, dem zentralen Terminus in Gramscis Denken. Die dialektische Anlage des Hegemoniebegriffs reflektiert die Bedingungen des Aufstiegs, Erhalts und Untergangs eines Herrschaftssystems (ebd.).

Die potenzielle Widerstandskraft subalterner Gruppen, die sich durch eine radikale Heterogenität gegenüber den dominanten Gruppen auszeichnet, wird in die Reflexionen der Historiker*innen der South Asian Subaltern Studies Group integriert und in postkolonialen Fragestellungen kontextualisiert (Guha 1983). Unter Zuhilfenahme des Konzepts der Subalternität kann das asymmetrische Herrschaftsverhältnis zwischen Kolonisatoren und Kolonisierten in der postkolonialen Theoriebildung anschaulich nachvollzogen werden. Spivak übernimmt den relational gemeinten Begriff und versteht ihn ebenfalls als Gegenbegriff zur Hegemonie. Sie sieht Subalterne jedoch nicht gänzlich als „Widerstandssubjekte“ mit eindeutigen Absichten und warnt vor Romantisierungen jeglicher Art (Spivak 1988: 197). Ihre Ausführungen verdeutlichen die doppelte Verletzlichkeit subalterner Frauen: Die Diskriminierung durch patriarchale Strukturen wird um die 
Dimension der wirtschaftlichen Ausbeutung infolge (neo-)kolonialer Dynamiken ergänzt. Mittels dekonstruktiver Strategien und marxistisch-feministischer Konzepte verfolgt Spivak in ihren literarischen Analysen vermeintliche Nebenschauplätze, um eine „Route des Verschweigens“ aufzudecken, die zeigt, wie die subalterne Frau systematisch aus der Haupthandlung ausgegrenzt wird (Castro Varela/Dhawan 2015: 160). Vermittels der Verflechtung postkolonialer und feministischer Perspektiven wird der eurozentrische und hegemoniale Diskurs, der Frauen aus der „Dritten Welt“ durch heterosexistische und rassistische Strukturen unterwirft, ,,doppelt kritisiert“ (Dhawan 2016: 172-173).

Literatur bildet für Spivak den rhetorischen Gegen-Ort, in dem die Narrationen subalterner Frauen Ausdruck finden können (Castro Varela/Dhawan 2015: 160). Ihre dekonstruktive Art des Lesens und Reflektierens fiktionaler Texte erschafft eine Form der Artikulationsmöglichkeit für Subalterne im postkolonialen Indien, die diese dazu befähigen soll, gegen ihre eigene Desintegration - etwa die Blockierung ihrer Teilnahme an demokratischen Prozessen zu protestieren und damit das Versagen der Dekolonisierung zum Ausdruck zu bringen (ebd.). Die subalterne Haltung wird genau dann zu einer theoretischen Fiktion mit hoher strategischer Bedeutung, wenn mit ihrer Hilfe das dominante koloniale Gerüst erschüttert werden kann (Spivak 1988: 204). In ihrer Auseinandersetzung mit der Stimmlosigkeit Subalterner aus dem genannten Essay (1988) kritisiert sie Michel Foucaults und Gille Deleuzes Positionen aufgrund ausgeklammerter Dimensionen globaler Arbeitsverteilung, makropolitischer Auseinandersetzungen und ideologietheoretischer Annahmen als unbeabsichtigt eurozentrisch (1988: $67 \mathrm{f}$.). Wenn etwa einige westliche Intellektuelle behaupten, die Masse der Subalternen könne für sich selbst sprechen, so werden spezifische Aspekte kolonialer Machtdiskurse ausgeblendet. Spivak unterstellt diesen Denker*innen eine essentialistische Agenda, die unweigerlich mit der Distanzierung von Verantwortlichkeit jeglicher Art einhergeht (Dhawan 2016: 180). Mit Marx' Der achtzehnte Brumaire des Louis Bonaparte, in dem französische Parzellenbauern als eine Gruppe nachgezeichnet werden, die keine kohärente Klasse bilden und daher durch eine bevollmächtigte Person repräsentiert werden müssen, unterscheidet sie zwischen Repräsentation als Darstellen und als Vertreten (Spivak 1988: 71). Darstellen meint in diesem Kontext „sprechen von"; Repräsentation als Vertreten hingegen bedeutet „sprechen für" (Dhawan 2016: 180). Diese beiden Ebenen fallen laut Spivak bei Foucault und Deleuze in bedenklicher Weise zusammen. Infolgedessen bildet sich ein vermeintlich kohärentes politisches Subjekt (ebd.). Spivak verdeutlicht, dass subalterne Handlungsmächtigkeit, vor allem die Gesinnung, sich selbst als Autor*in 
eigener Repräsentation in einem hegemonialen System zu verstehen, letzten Endes zum Scheitern verurteilt ist (Castro Varela/Dhawan 2015: 198).

„Subalterner Aufstand [...] ist ein Bemühen, sich selbst in die Repräsentation einzubringen, und zwar nicht entlang der Linien, die von den offiziellen institutionellen Repräsentationsstrukturen vorgegeben werden. Zumeist erreicht er nichts. Das ist das Moment, das ich ,nicht sprechen“ nenne" (Spivak 2008: 144, zit. in Münker/ Roesler 2012: 190).

Ursache der Sprachlosigkeit ist das hegemoniale System, das jene Ungleichheitsverhältnisse permanent (re-)produziert, etwa durch eine koloniale Geschichtsschreibung und dominierende Geschlechterkonstruktionen. Spivak veranschaulicht die Produktion der Sprachlosigkeit Subalterner anhand konkreter Beispiele, die nahelegen, dass kein rhetorischer Raum existiert, in dem Subalterne aus dem globalen Süden im Westen sprechen könnten - und dies, obwohl in westlichen Staaten wesentliche Entscheidungen getroffen werden, die das Leben der Subalternen direkt und indirekt beeinflussen (ebd.: 180). Selbst wenn sie es versuchten, würden sie nicht gehört werden (Spivak 1996: 292), weil auch das Hören hegemonial strukturiert ist (Castro Varela/Dhawan 2015: 199). Die sogenannte „epistemische Gewalt“ (epistemic violence) hat ihren Ursprung nicht immer im Kolonialismus, sondern wird in neokolonialen Machtverhältnissen, „die schließlich das postkoloniale Subjekt herstellen“ (ebd.: 183), fortgesetzt (Spivak 1993: 211). Unter epistemischer Gewalt versteht Spivak die Macht, zu bezeichnen und damit den Versuch, Erfahrungen, Perspektiven und Kämpfe von Minderheiten in abstrakten Überbegriffen „einzufrieren“ (Castro Varela/Dhawan 2015: 184), beispielsweise die Arbeiter, die Frauen oder die Kolonisierten (ebd.). Im Unterschied zu anderen Kolonialismusanalysen implizieren Spivaks Überlegungen neokoloniale Konstellationen. Sie führt in diesem Zusammenhang Indigene aus Indien an, die im Namen von Fortschritt und Entwicklung am untersten Ende der sozialen Hierarchie verortet werden, indem sie systematisch ausgebeutet werden (ebd.: 183). Die Betroffenen schauen derweil zu, wie ihr Leben vom westlichen Ideal neoliberal-kapitalistischer Dynamiken, das auf ,ferngesteuertem Leiden“ basiert, zerstört wird (Spivak 1996: 277). Die Überwindung fundamentaler Machtasymmetrien sowie ökonomischer und soziopolitischer Ungleichheiten kann laut Spivak nicht kurzerhand durch die Gewährleistung von Grundbedürfnissen der Menschen aus der „Dritten Welt“ bewerkstelligt werden. Für jene, die durch Gender-, Klassen- und Kastensysteme brutal ausgebeutet werden, sind die Rituale der Demokratie nichts anderes ,als ein absurdes Theater" (ebd.: 79). Die erwähnte Überwindung asymmetrischer Beziehungen 
muss daher mittels einer „Neuordnung von Begehren“ (Spivak 2009: 36) auf beiden Seiten der Postkolonialität - der Subalternen sowie der privilegierten Klassen - herangebildet werden (Dhawan 2016: 191).

Vor diesem Hintergrund wird in der vorliegenden Arbeit nicht der Anspruch erhoben, für geflüchtete Menschen $\mathrm{zu}$ sprechen, es wird aber auch nicht behauptet, dass man gar nicht für sie zu sprechen brauche, weil sie ohnehin nicht gehört würden. Vielmehr wird der Versuch unternommen, im Sprechen $z u$ afghanischen Individuen die eigene Position mitzureflektieren, ohne sie zu verstecken (vgl. Münker/Roesler 2012: 190). Dies geschieht auf der Grundlage empirisch erhobener Daten und aus einer männlichen Perspektive. Dabei geht es nicht um die marginalisierte Sicht von Fluchtmigrierenden per se, sondern um das Aufdecken heterogener Subjektpositionen (Lebenswelten) innerhalb der Institution Asyl. Im Zuge dessen wirkt sich zwar die persönliche Interpretation des Autors auf die Artikulation jener Positionen aus und beeinflusst die Repräsentation maßgeblich. Dennoch lässt sich dadurch ein gewisser rhetorischer Raum schaffen, der es in geringem Umfang ermöglicht, nicht gehörte Geschichten, Erfahrungen und Gefühle afghanischer Individuen zu artikulieren.

\subsection{Homi K. Bhabha: Identitäts- und kulturtheoretische Überlegungen}

Der parsische Literaturwissenschaftler und Kulturtheoretiker Homi K. Bhabha (1949* in Bombay) gilt neben Edward W. Said (1935-2003) und Gayatri Chakravorty Spivak als einer der einflussreichsten postkolonialen Denker unserer Zeit (Struve 2013: 9). Als „,[W]eder Hindu noch Muslim“ (Bhabha 2012: 53), interessieren ihn die spannungsreichen Positionierungen zwischen den Kulturen, in denen komplexe Konfigurationen Differenz und Identität, Vergangenheit und Gegenwart, Innen und Außen, Einbeziehung und Ausgrenzung erzeugen (Bhabha 2000: 1). Nach seinem MA-Abschluss in Oxford promovierte er 1990 über das literarische Werk des postkolonialen Autors und Nobelpreisträgers V. S. Naipaul und erhielt 1994 die Professur für englische Literatur an der Universität von Chicago (Struve 2013: 11). Seit 2001 hat er die Anne-F.-Rothenberg-Professur für Humanities und die Leitung des Humanities Center an der renommierten Harvard-Universität inne (ebd.). Die literatur-, kultur- und identitätstheoretischen Überlegungen aus Nation and Narration (1990) und The Location of Culture (1994; dt. Übersetzung: 2000) mit ihren Ideen, Konzepten, Argumentations- und Denkfiguren haben enorme Auswirkungen auf die Kultur- und Sozialwissenschaften (Göhlich 2010: 316). Als einer der Hauptvertreter des ,postcolonial 
turn“ (Bachmann-Medick 2006: 189) reflektiert Bhabha als Migrant seine spannungsreichen Erfahrungen mit und über Migration, die er stets als Erfahrungen zwischen den etablierten Positionen erlebte (Struve 2013: 9). Er versteht seine Ansätze als eine Intervention in die ideologischen Diskurse und Machtverhältnisse in der „westlichen Moderne“ (Bhabha 1994: 239), die den

„oft von Benachteiligung gekennzeichneten Geschichten von Nationen, Ethnien, Gemeinschaften und Völkern eine hegemoniale ,Normalität' [...] verleihen. Sie formulieren ihre kritischen Revisionen im Umkreis von Fragen der kulturellen Differenz, der sozialen Autorität und der politischen Diskriminierung [...]“ (Bhabha 2000: 255).

Zentrales Thema der Kernkonzepte Bhabhas ist die Produktion von Differenz in einem Raum „dazwischen“ (Bonz/Struve 2006: 141). Er verortet Kultur in „Zwischenräumen“ und „Bereichen des darüber hinaus“ (beyond), die aus Überlappungen und Verschiebungen von Differenzbereichen hervorgehen (Göhlich 2010: 317). Mit der Differenzproduktion oder -verhandlung ist die Dynamik gemeint, die sich im Zuge der Abwendung von generalisierenden Kategorien wie Geschlecht, Hautfarbe und Klasse über eine bewusstere Wahrnehmung des Subjekts, seiner Position und seiner Narrative hinaus auf die Artikulation von Momenten kultureller Differenz konzentriert (ebd.). Kulturverortende und generierende Differenzverhandlungen sind für Bhabha unter anderem in künstlerischen Werken zu finden, wobei er sich theoretisch größtenteils von dem bedeutenden französischen Psychoanalytiker, Schriftsteller und Vordenker der Entkolonialisierung Frantz Fanon (1925-1961) beeinflussen lässt. So rücken wie von selbst drei zentrale und kontroverse Topoi unserer Zeit in den Vordergrund seiner Untersuchungen: Grenzen, Differenzen und Übergänge (Bhabha 2007: 29). Diese großen gesellschaftspolitischen Bereiche, gleichsam rhetorische Figuren für Bhabha, bilden die Eckpfeiler seines kultur- und identitätstheoretischen Ansatzes. Seine abstrakte, mit vielen rhetorischen Finessen und komplexen Gedanken angereicherte Sprache (Bachmann-Medick 1998: 23) wird teils als „mystisch“ und „geheimnisvoll“ (Moore-Gilbert 1997: 115) kritisiert. Drei Hauptbegrifflichkeiten seiner Kultur- und Identitätstheorie sind „Mimikry“, „Hybridität“ und „Dritter Raum“. Für den Kontext dieser Studie ist dabei besonders die Erörterung und Bestimmung der Begriffe Identität und Kultur von Relevanz. 


\section{Identität und Kultur}

Identität bildet bei Bhabha keine monolithische Einheit im Sinne einer fixen sozialen Rolle, einer habituellen Prägung, einer performativen Leistung, eines kognitiven Selbstbildes, eines konstruierten Narrativs usw. Sie erscheint vielmehr als besonders differenzierte und reflexive Identität, deren Ausgang stets die Differenz bildet, die im Dritten Raum ständig (re-)produziert wird (Göhlich 2010: 326). Ähnlich wie Bhabhas Denkweise nicht von der Kultur und dem Subjekt ausgeht, bedeutet Identität gleichermaßen eine fragmentarische Produktion von Sinn und Wert (Bhabha 2000: 256). In dieser Perspektive gibt es daher keinen Platz für Theorien des kulturellen Relativismus oder Pluralismus (ebd.: 258). Sein semiotisch-diskursives Kulturverständnis fasst Kultur als bedeutungsgenerierendes System auf. Eine Kultur ist nicht durch ihre Inhalte, beispielsweise Werte, Rituale und Gebräuche, zu kennzeichnen. Sie funktioniert eher als Struktur der Symbolisierung (Struve 2013: 43). Kultur wird als ungleichmäßige, unvollendete Produktion von Bedeutung und Wert verstanden, die sich häufig aus nicht miteinander zu vereinbarenden Prinzipien konstituiert (Bhabha 2000: 256). Kulturen sind bedeutungsgenerierende Prozesse und Subjekte stiftende Praktiken, die sich gerade durch ihre Widersprüchlichkeit hinsichtlich normativer Werte, Narrative und Machtansprüche auszeichnen (Struve 2013: 43). Die konkurrierenden Wert- und Bedeutungssysteme sind existenziell für das Subjekt und deshalb mit Gefahren verbunden (ebd.). Kultur wird zur Überlebensstrategie für das Subjekt (Bhabha 2000: 257), dessen Handlungsmacht außerhalb der Diskurse des Individualismus liegt (ebd.: 278). Bhabhas Blick für das Prozesshafte, Dynamische und seine Affinität für die Räume „dazwischen“ erschweren einerseits den Zugang zu seiner Theorie, andererseits offenbaren sich gerade hier grundlegende anti-essentialistische Momente seines Denkens, die der kulturellen Differenz und der Ambivalenz (Struve 2013: 50). Diese spannungsvolle Differenz zwischen Kulturen verursacht eine Störung, die Verunsicherung aufkeimen lässt und einen mehrdeutigen Zwischenraum öffnet. Das „Andere“ entsteht demnach nicht bloß durch eine Bewegung der Abgrenzung, sondern durch innere Spaltung und Uneindeutigkeit angesichts der entstandenen Ambivalenz (ebd. 51). Um dies besser nachzuvollziehen, bedarf es der Erläuterung des Konzepts der Hybridität oder Hybridisierung - im Folgenden synonym verwendet -, das im Diskursverständnis Bhabhas wurzelt. Für den Literaturwissenschaftler Bhabha steht der Diskurs, also all die (kolonialen) literarischen und sprachlichen Produktionen und Prozesse, im Vordergrund seiner Überlegungen (Göhlich 2010: 323). Mit seiner spezifischen Art der dekonstruierenden Diskursanalyse bildet er das Konzept der Hybridität aus, das er erst hinterher auf die Kultur und Identität anwendet (ebd.). Er geht von einer grundsätzlich binären Performativität englischer Literatur im 
kolonialen Raum aus, die maßgeblich dazu beiträgt, die Wirklichkeit zu spalten und in sich zu verrücken:

„Die Ausübung der kolonialistischen Autorität erfordert jedoch die Produktion von Differenzierungen, Individuationen und Identitätseffekten, mittels derer Bevölkerungen qua diskriminatorischer Praktiken als Untertanen definiert werden können, die die sichtbare und transparente Markierung der Macht an sich tragen. Eine derartige Form der Unterwerfung/Subjektbildung (subjection) ist etwas anderes als das, was Foucault als ,Macht durch Transparenz" beschreibt [...]. Der ,Teil' (der immer der kolonialistische Fremdkörper sein muss) muss das ,Ganze' (eroberte Land) repräsentieren, aber dieses Repräsentationsrecht basiert auf der radikalen Differenz des Teils. Solches Zwiedenken kann nur aufgrund der soeben beschriebenen Strategie der Verleugnung funktionieren, die von uns eine Theorie der ,Hybridisierung ' von Diskurs und Macht erfordert, die wiederum von den Theoretikern, die sich mit dem Kampf um ,Macht ' beschäftigen, dies aber lediglich als Puristen der Differenz tun, leider außer acht gelassen wird“ (Bhabha 2000: 164).

Seine Untersuchungen stellen die erwähnte zwiespältige Natur des westlichen Denkens in der Moderne infrage und gehen der ihr inhärenten kolonialistischen Logik nach. Bhabhas theoretische Ausrichtung unterscheidet sich deutlich von anderen postkolonialistischen Theoretiker*innen, die die defekte Denkweise als Motor des Kolonialismus verstehen und beschreiben, wie etwa Gayatri Chakravorty Spivak, die dies mit ihrem Konzept des othering (1985) anschaulich zu tun verstand. Es ist die erwähnte fundamentale Spaltung ,,between the mother culture and its bastards, the self and its doubles" (Bhabha 1994: 111), welche die Unterwerfung des „Anderen“ bewirken. Hybridität bzw. Hybridisierung meint demgemäß bezogen auf die Ebene der Kultur jenen Bereich und das Ergebnis von Kulturkontakt, bei dem Vermischungen auch Sinn- und Machtverschiebungen bedeuten (Struve 2013: 100). Der Hybridisierungsprozess ist von anderen Ansätzen und Vorstellungen zu unterscheiden, die den Vermischungsprozess als eine Art Synthese im Sinne eines harmonischen Ausgleichs zwischen gegensätzlichen Kulturen verstanden wissen wollen (ebd.: 101). Hybridisierung wirkt durch ihre Diskursivität vielmehr innerhalb der betroffenen Kulturen und nicht zwischen zwei differenten Kulturen (Struve 2013: 103). Sie ist kein „multikultureller Schmelztiegel“, in dem sich alle Differenzen letztlich in einem dialektischen, erlösenden Spiel der Erkenntnis auflösen, sondern vermischte Unauflöslichkeit (ebd.), die durch Verunsicherung bei allen Beteiligten des kulturellen Kontaktes stattfindet (ebd.: 112). Hybridität wird als Zeichen der Produktivität kolonialer Macht begriffen, eine Art strategischer Umkehrung des Prozesses der Beherrschung unterdrückter Menschen durch Verleugnung. Die Autorität kolonialer Macht ist für Bhabha nämlich nicht bloß „gegeben“ - wie 
etwa für Edward Said -, sondern erlangt ihre Stärke erst durch einen Prozess der Verleugnung seitens der Unterdrücker (Münker/Roesler 2012: 191). Mithilfe der Produktion diskriminatorischer Identitäten von Minderheiten soll eine ,ursprüngliche“ Identität der Autorität sichergestellt werden (Bhabha 2000: 163). Hybridität deformiert und deplatziert diese Form der Diskriminierung und Beherrschung. Indem sie die Wirkung der Verleugnung im gesamten Machtgefüge umkehrt und durcheinanderbringt, wirkt sie subversiv (Münker/Roesler 2012: 191). Dabei betont Bhabha die Neuartigkeit des Ergebnisses der Durchmischung zweier Positionen:

„The process of cultural hybridity gives rise to something different, something new and unrecognisable, a new area of negotiation of meaning and representation“ (Rutherford/Bhabha 1990: 211).

Somit entsteht ein neuer Raum, der Dritte Raum, in dem die Neukonstruktionen neue Formen voller Ambivalenzen und Differenzen annehmen (Bonz/Struve 2006: 144), ohne dabei Hierarchien zu übernehmen (Bhabha 2000: 5). Hybridität funktioniert als zwischenräumlicher Übergang zwischen Identifikationen. Damit ist die agency der Subjekte angesprochen, deren sich stetig wandelnde Art des Ausdrucks kultureller Praktiken in einem sich verändernden Kontext niemals das Gleiche bedeuten kann, sondern immer eine Differenz mit sich führt (Sieber 2012: 100). Hybridität stellt die prozessuale und kreative Neukonstruktion von Identitäten dar und wird $\mathrm{zu}$ einem zentralen Merkmal und Leitbegriff von Bhabhas Texten. Um diesen abstrakten und schwer zu fassenden Begriff zu konkretisieren, greift er auf die räumliche Semantik zurück, die bereits in Wendungen zur Erklärung des Hybriditätskonzepts sowie als Denkfiguren der Differenz, wie „dazwischen“" und „beyond“, zum Ausdruck kommen (Struve 2013: 121). Das Konzept des Dritten Raumes, dessen Namen er sich von dem marxistischen Literaturwissenschaftler Fredric Jameson (1934*) borgt (Bhabha 2000: 217) und dessen grundlegende Inspiration durch eine Installation der bildenden Künstlerin Reneé Green mit dem Titel Sites of Genealogy erfolgte, meint eine nicht zu fixierende räumlich semantisierte Kategorie (Struve 2013: 123). Gemeint ist mit dem Begriff ein instabiler, höchst ambivalenter Raum, in dem agency performiert, konstituiert und verhandelt wird. Letzteres rüttelt aufgrund seiner unechten Natur an der kolonialen Autorität (Göhlich 2010: 327). Der Dritte Raum als Konzeptmetapher von Hybridität kombiniert die Ideen von Prozessualität, Hybridisierung und kultureller Zeitlichkeit und bildet somit eine paradigmatische Bhabha'sche Kategorie (Bonz/Struve 2006: 145). Die Liminalität des Dritten Raums verrückt Fixierungen, hebt sie auf und ermöglicht 
damit eine Neuverteilung der agency (Göhlich 2010: 328). Der Dritte Raum ist die Vorbedingung für die Artikulation kultureller Differenzen, die es ermöglicht, Kulturen nicht als festgelegt und einheitlich zu betrachten. Der Dritte Raum ermöglicht die Neukonfiguration der Struktur von Bedeutung und Referenz zu einem ambivalenten Prozess, der den Spiegel der Repräsentation zerstört (Bhabha 2000: 56). Mithilfe der Denkfigur des Dritten Raums wird ersichtlich, dass kulturelle Vorstellungen und Systeme stets in einem ambivalenten und widersprüchlichen Äußerungsraum konstruiert werden (ebd.: 57). Implizite oder explizite Ansprüche auf „Reinheit“ von Kulturen und Identitäten sind aus diesem Grund nicht tragbar (ebd.). Die Sinn- und Bedeutungsverschiebungen des Dritten Raums bringen eine Differenz hervor, die die Mimikry ausmacht - ,fast dasselbe, aber nicht weiß “ (Bhabha 2000: 132). Während Hybridität und Dritter Raum eher als Kategorien zu verstehen sind, die kulturelle Mischprozesse als Phänomene zu beschreiben und zu untersuchen erlauben, bildet Mimikry eine Strategie der Hybridität (Struve 2013: 131). Sie bezeichnet eine schützende Anpassung der unterdrückten Gruppe durch eine unvollständige Imitation der Kolonisatoren und bildet so eine „Metonymie der Präsenz“ (Bhabha 2000: 134). Der Begriff der Mimikry beruht dabei auf Bhabhas Übersetzungsbegriff. Übersetzung bedeutet die Distanz oder besser Entfremdung zwischen Signifikant (Bezeichnendes) und Signifikat (Bezeichnetes), die Bhabha als Re-Signifikation, also als Neu-(Be-) Deutung in der Bewegung der Bezugnahme auf bestehende Zeichen versteht (Bonz/Struve 2006: 146).

„(T)ranslation is also a way of imitating, but in a mischevious, displacing sense imitating an original in such a way that the priority of the original is not reinforced but by the very fact that it can be simulated, copied, transferred, transformed, made into a simulacrum and so on: the original is never finished or complete in itself" (Bhabha/Rutherford 1990: 210).

Als berühmtes Beispiel für diese Re-Signifikation, dient der Roman Die Satanischen Verse von Salman Rushdie. Dessen Auslegung der Korandebatte, die in einer anderen Kultur erfolgt, misst den mit dem Koran assoziierten Signifikanten andere Bedeutungen $\mathrm{zu}$ als im ursprünglichen, iranischen Kontext. Mittels Übersetzungsprozessen entstehen hier Selbstentfremdungseffekte, die zu Hybridisierungen führen (Struve 2013: 131), was letztlich die Autorität der Kolonisatoren unterminiert, weil die Übersetzung nicht korrekt ist, selbst hybrid wird und somit das ursprüngliche Wort ,infiziert“; Übersetzung bedeutet für Bhabha demnach ein unaufhörlicher Kampf um Identitäten, der Machthierarchien hinterfragt (ebd.). Auf die Kultur bezogen, reformuliert er das aus der 
Biologie stammende Prinzip der Mimikry als Kategorie zur Deskription kultureller Differenz (Bonz/Struve 2006: 149). Anpassungsstrategien der Mimikry sind gemäß Bhabha „one of the most elusive and effective strategies of colonial power and knowledge" (1994: 85). Mimikry entsteht dabei als Repräsentation der Differenz. „Mimikry ist also das Zeichen einer doppelten Artikulation, eine komplexe Strategie der Reform, Regulierung und Disziplin, die sich den Anderen ,aneignet ' [...], indem sie die Macht visualisiert" (Bhabha 2015: 114). Sie schafft Diskontinuitäten und wirkt so aufgrund ihrer doppelten Natur bedrohlich. Das Bedrohliche an ihr ist nicht, dass sie zu verbergen oder zu vertuschen sucht, sondern die „doppelte Sicht“, die aus ihr hervorgeht (Bhabha 2000: 130). Bhabha zieht in seinen Überlegungen den französischen Psychoanalytiker Jacques Lacan (1901-1981) heran. Mimikry sei demnach eine Form von Ähnlichkeit, die sich von der Präsenz dadurch unterscheidet (oder sie dadurch abwehrt), dass sie teils metonymisch präsentiert wird (ebd.: 133). Die Repräsentation von Sinn und Identität wird somit neu ausbuchstabiert, wobei sich zahllose neue Kombinationen von Bedeutungen ergeben, so dass nichts mehr dingfest zu machen ist, weil keine Essenz verborgen wird (ebd.). Es geht nicht um die Heranbildung einer eigenständigen, präsenten Identität, sondern um das Formulieren, das Repräsentieren und die Performativität selbst (Bonz/ Struve 2006: 149). Lacans Annahme, das Unbewusste resultiere aus der Sprache und funktioniere wie diese, ist für Bhabha deshalb so bedeutsam, weil sich erst auf dieser Basis der Subjektwerdungsprozess vollziehen kann. In seinem Aufsatz „Spiegelstadium“ (Lacan 1936/49) unterscheidet Lacan zwischen zwei Ich-Instanzen, die sich in der französischen Sprache durch moi und je ergeben, und fokussiert jene Phase der Menschwerdung, in der das Ich sich durch die Begegnung mit seinem externen Ebenbild - dem Anderen - konstituiert (Müller-Funk 2016: 176). Das menschliche Subjekt ist unaufhebbar gespalten, weil das betrachtende Individuum niemals mit seinem imaginären Spiegelbild identisch sein wird. „Ich“ impliziert unter diesem Gesichtspunkt stets eine Projektion. Lacans radikale Weiterführung des prominenten Zeichenmodells von Ferdinand de Saussure (1857-1913) mündet letztlich ganz im Sinne des Poststrukturalismus in einer sich niemals abschließenden Bewegung des Identifikationsprozesses. Bhabha verwendet genau diesen Ansatz, um die identifikatorische Instabilität des Dritten Raums, die keine fixierte kulturelle Differenz mehr zwischen „Eigenem“ und „Anderem“ zulässt, nachzuzeichnen (Struve 2013: 23-24). Aus diesem Grund belegen westliche Theoretiker*innen laut Bhabha „die Anderen“ mit Bildern und Metaphern, um sie beschreiben und somit eindämmen und kontrollieren zu können (Struve 2013: 33). Durch diese Eindämmung und die mit ihr einhergehende kulturelle Politik der Differenz werden 
„Andere“ in der wissenschaftlichen Debatte nivelliert, indem die aus ihr hervorgehenden Narrative zum geschlossenen Interpretationszirkel werden (Bhabha 2000: 48).

\begin{abstract}
„Das Andere verliert seine Macht, zu signifizieren, zu negieren, sein historisches Begehren ins Spiel zu bringen, seinen eigenen institutionellen und oppositionellen Diskurs zu etablieren. Ganz gleich, wie untadelig das Wissen über den Inhalt einer , anderen' Kultur sein mag, ganz gleich, wie antiethnozentrisch sie repräsentiert wird: ihre Verortung als Abschluss großer Theorien und die Forderung, dass sie in analytischer Hinsicht immer das gute Objekt der Erkenntnis, die gefügige Summe der Differenz zu sein hat, reproduzieren eine Beziehung der Herrschaft über sie und stellen somit die schwerwiegendste Anklage der institutionellen Macht kritischer Theorie dar." (Bhabha 2000: 48)
\end{abstract}

Nichtsdestotrotz verteidigt Bhabha seine fortlaufende Referenzierung auf den „Anderen“ unter Verweis auf die innovative Kraft der Ansätze von Lacan und Foucault (ebd.). Er zielt damit auf einen theoretischen Ansatz ab, der sich selbst zu übersetzen imstande ist und damit das Projekt der Moderne von Grund auf infrage stellt (ebd.: 49).

\title{
4.6 Judith Butler: Subjektivierungstheoretische Überlegungen
}

Wurden in den vorangehenden Kapiteln postkoloniale Sichtweisen skizziert, die sowohl Macht- und Hierarchieverhältnisse als auch die radikale Pluralisierung der globalisierten Welt berücksichtigen, wird nun mit Judith Butler die Subjektwerdung (Subjektivation) unter postmodernen Bedingungen reflektiert. Mithilfe dieser Überlegungen soll zum einen der Zusammenhang zwischen Macht, Subjektbildung und Emotionen im Asylprozess und zum anderen der ambivalente Prozess der Unterwerfung differenzierter nachvollzogen werden.

Die US-amerikanische Philosophin und Philologin Judith Butler (1956*), seit 1994 Professorin für Rhetorik und Komparatistik an der University of California, Berkeley, untersucht in ihrer Theorie der Subjektivation das komplexe Verhältnis von Subjekt und Macht. Besonders in ihrer Arbeit Psyche der Macht. Das Subjekt der Unterwerfung (2001) denkt sie in Übereinstimmung mit Bhabha nicht in essentialistischen, kulturalistischen oder ethnisierenden Reduktionen (Kleiner/ Rose 2014b: 9). Zentrales Anliegen Butlers, deren Arbeiten dem Poststrukturalismus zuzuordnen sind, ist die Erweiterung der Identitätsbegriffe, die das Ausgeschlossene und Verworfene miteinbeziehen. In ihren Ausführungen kombiniert 
sie gesellschaftliche, diskursive und psychologische Mechanismen im Anschluss an Foucault (Schneider 2018: 77). Der Begriff der Subjektivation schließt an Foucaults Terminus assujetissement (Butler 2001: 21) an und meint die Bedingungen dafür, dass Menschen überhaupt zu Subjekten (gemacht) werden.

Butler beginnt ihre Reflexionen mit der Erkenntnis, dass sprachliche Grammatik grundsätzlich begrenzend wirkt. Sie suggeriert damit, dass bereits vor der Machteinwirkung ein Subjekt existiert: „Grammatisch betrachtet scheint es zunächst ein Subjekt geben zu müssen, das sich auf sich selbst zurückwendet; ich werde jedoch die Auffassung vertreten, dass es ein solches Subjekt nur als Folge ebendieser Reflexivität gibt“" (ebd.: 68). Die genannte Reflexivität spiegelt sich in der Zirkularität der Subjektivation wider. Butler reflektiert in Psyche der Macht zunächst klassische Positionen der Subjektwerdung bei Hegel, Nietzsche, Marx, Freud und Althusser, die ihrerseits Varianten der Zirkularität im Prozess der Subjektwerdung nachzeichnen. Macht ist, wie Butler zusammenfasst, auf ein vorgängiges Schuldgefühl ihrer Subjekte angewiesen; doch können die klassischen Ansätze nicht hinreichend erklären, wie es zu der charakteristischen Wendung des Subjekts gegen sich selbst kommt (Redecker 2011: 96). Für Butler ist diese Wendung gegen sich selbst das, was das Subjekt ausmacht. Daher fragt sie nach der Entstehung von Subjekten, bevor sie formiert werden (Butler 2001: 111). Sie möchte über den Unterwerfungsakt hinaus verstehen, weshalb Subjekte an der eigenen Unterwerfung haften bleiben (ebd.: 98): „Wenn Unterwerfung also eine Bedingung der Subjektwerdung ist, stellt sich die Frage: Welche psychische Form nimmt die Macht an?"“ (ebd.: 8)

Zur Beantwortung dieser zentralen Frage werden zwei Aspekte angeführt. Zum einen handelt es sich um ein fundamental menschliches Existenzstreben, das Butler mit Bezug auf Spinozas „conatus essendi“15 als „Begehren zu existieren“ erklärt. Dieses Begehren gleicht der Begierde nach sozialer Anerkennung gemäß Alexandre Kojève (siehe nächstes Unterkapitel), doch spricht Butler noch nicht von der normativ aufgeladenen Kategorie der Anerkennung. Sie hebt vielmehr die Wahllosigkeit und die damit verbundene Ohnmacht des Existenzbegehrens hervor, indem sie den anfänglichen Zustand der Subjektwerdung mit der Machtlosigkeit eines Säuglings vergleicht, der selbst für die grausamste Bezugsperson eine Anhaftung und Zuneigung entwickeln muss, um überhaupt weiterzuleben

15 ,Jedes Ding strebt gemäß der ihm eigenen Natur, in seinem Sein zu verharren.“ Lehrsatz 6, Teil III der Ethik in geometrischer Ordnung (Spinoza 2015: 219). Judith Butler zieht Spinozas Interpretation des Conatus aus seinem zitierten Hauptwerk ebenfalls in ihren anderen Werken heran (Butler 2009: 56; 2003: 58 f.) 
(2001: 13). Wie das Kind des bloßen Überlebens wegen Liebe für seine Bezugsperson(en) hervorzubringen genötigt ist, so ist das Subjekt gezwungen, sich an soziale Normen seiner Lebenswelt zu binden. Die begehrende Haltung des Kindes behält das Subjekt im weiteren Verlauf des Lebens bei und grenzt sich zugleich von dieser Abhängigkeit ab. In der Abgrenzung sieht Butler eine Verleugnung, die sie als maßgeblich für die charakteristische Wendung des Subjekts gegen sich selbst begreift.

\begin{abstract}
„Ohne diese in Abhängigkeit ausgebildete Bindung kann kein Subjekt entstehen, aber ebenso wenig kann irgendein Subjekt es sich leisten, dies im Verlauf seiner Formierung vollständig zu ,sehen'. Das Verhaftetsein in seinen ursprünglichen Formen muss sowohl entstehen wie verleugnet werden, seine Entstehung muss seine teilweise Verleugnung sein, soll es überhaupt zur Subjektwerdung kommen“ (ebd.).
\end{abstract}

Der Bereich sozialer Normen und Identifikationsmöglichkeiten konstituiert den zweiten Gesichtspunkt ihrer Antwort auf die Frage nach der psychischen Form von Macht. Das Feld des Normativen gibt vor, welche Art des Begehrens akzeptabel ist und bestimmt zugleich, welche identitären Positionen unerwünscht sind. Damit sind gesellschaftliche Tabus gemeint, die das Subjekt von Anfang an bedrohen (Redecker 2011: 98) und sich je nach sozialem Umfeld in ihren Ausmaßen unterscheiden. Das Subjekt, das sich nicht mit vorgegebenen Begehrensformen identifizieren kann, wird im Feld der Macht genötigt, spezifische, den gesellschaftlichen Normen entgegengesetzte Identitäten - etwa identitäre Positionen, die dem heteronormativen Ideal widersprechen $-\mathrm{zu}$ verwerfen. Da das Feld der sozialen Normen von Ausschlüssen bestimmter Begehrens- und Identitätsformen durchdrungen ist, schreibt es dem begehrenden Menschen auch Verluste zu (ebd.: 99). Die Unterwerfung des Individuums geht demzufolge immer mit der Verwerfung bestimmter Formen und Inhalte des Begehrens einher. Infolge dieser Verwerfungen entsteht im nunmehr gespaltenen Subjekt eine Melancholie, die gleichzeitig die Grenzen seiner Reflexivität markiert (Butler 2001: 28). Soziale und kulturelle Normen legen letztlich fest, was eine anerkennungsfähige Existenz ist oder wie „Verständlichkeit im kulturellen Feld erfüllt"“ (Kämpf 2006: 251) wird.

In diesem Verständnis ist die Subjektwerdung nicht ein linearer Prozess einer Verinnerlichung gesellschaftlicher Erwartungen; Subjektwerdung bezeichnet vielmehr den ambivalenten Prozess des Unterworfenseins durch Macht und zugleich der aus ihm folgenden Handlungsfähigkeit (Butler 2001: 8). Die Handlungsfähigkeit des Subjekts entsteht erst durch seine Unterwerfung unter die jeweiligen Machtverhältnisse. Einerseits wird der unterworfene Mensch als Effekt einer 
vorgängigen Macht und andererseits als Möglichkeitsbedingung für eine radikal bedingte Form der Handlungsmacht begriffen (ebd.: 19). Diese kraftvolle Ambivalenz im Subjekt konzeptualisiert Butler in Anlehnung an das Konzept der Interpellation oder Anrufung des französischen Philosophen Louis Althusser (1918-1990). Subjektivität wird demnach performativ erzeugt, indem ein Subjekt von einer Machtinstanz durch den Sprechakt angerufen wird. Unter Interpellation versteht Butler einen Prozess, der Subjekte durch Diskurse fixiert. Subjektivation markiert eine relationale und zirkulär angelegte Abhängigkeitsbeziehung von Unterwerfung und Formierung; dabei werden die diskursiv-unterwerfende Anrufung und die subjektive Positionierung gleichermaßen als produktiv verstanden (Jäckle 2015: 109). Indem eine Person auf den Ruf reagiert, unterwirft sie sich gleichsam der herrschenden diskursiven Ordnung. Die Anrufung wird in diesem Kontext als Aufforderung zu bestimmten Verhaltensweisen verstanden, die durch die erwähnten normativen Begehrensformen vorgegebenen werden. Abhängig von der jeweiligen kulturellen und soziopolitischen Norm variiert die Beschaffenheit der Interpellation, doch bleibt das Prinzip stets unverändert. Ein wesentliches Element der Anrufung besteht in der Wiederholung (Iterabilität):

„Das Foucaultsche Subjekt wird nie vollständig in der Unterwerfung konstituiert; es wird wiederholt in der Unterwerfung konstituiert, und es ist diese Möglichkeit einer gegen ihren Ursprung gewendeten Wiederholung, aus der die Unterwerfung so verstanden ihre unbeabsichtigte Macht bezieht“" (ebd.: 90).

Durch die wiederholte Performativität des Sprechakts der Anrufung wird nicht bloß eine Äußerung getroffen, sondern eine materielle Wirklichkeit erschaffen (Butler 1997: 22). Sprache ist für Butler immer mit einem performativen Effekt verknüpft. Die Wirksamkeit der Sprechakte liegt in letzter Konsequenz in der zitatförmigen Wiederholung von Konventionen und der mit ihnen assoziierten Autorität des Diskurses (Schneider 2018: 79). In dieser Perspektive erhält jede Person ihre spezifische Rolle und Position in der Gesellschaft dadurch, dass sie sie selbst mitkonstruiert. Weil das Subjekt weder gänzlich durch die anrufende Macht determiniert wird noch seinerseits die anrufende Macht vollständig determiniert (Butler 2001: 12), liegt Macht nicht außerhalb des Menschen. Entsprechend kann sie auch nicht ,gegen“ dessen Willen durchgesetzt werden (ebd.: 24).

Dieses Subjektverständnis unterscheidet sich durch die Betonung der inneren Zwiespältigkeit von klassischen Ansätzen, die noch in einfachen Dichotomien denken. Das Butler'sche Machtverständnis umgeht solche Dichotomien auf elegante Art und Weise. Die Triebfeder der Subjektivation, das Begehren, bildet 
die Basis der Verletzlichkeit des Subjekts, das genötigt ist, nach Anerkennung seiner eigenen Existenz ,in Kategorien, Begriffen und Namen zu trachten, die es nicht selbst hervorgebracht hat" (Butler 2001: 25). Das Individuum sucht also die Zeichen seiner Existenz außerhalb seiner selbst (ebd.). Der Umstand, dass wir anrufende und angerufene Wesen zugleich und damit von positiver oder negativer Anrufung anderer abhängig sind, trägt ebenfalls zur fundamentalen Vulnerabilität des Subjekts durch Sprache bei (ebd.: 44). Das Begehren nach Anerkennung platziert somit das Subjekt in eine Position der Vulnerabilität gegenüber der dominierenden Macht. Butler versteht unter dominierenden Machtstrukturen wirklichkeitsgenerierende gesellschaftliche Diskurse (Villa 2008: 149). Aus der Unterwerfung des Subjekts kann eine Verwerfung werden, die Gegenmacht in Form von Handlungsmächtigkeit entstehen lässt (ebd.: 99). Auch hier gilt der Grundsatz der Wiederholung, um Gegenmacht und Wirksamkeit zu festigen (Butler 2006: 198). Butler unterscheidet in Anlehnung an Foucault Missachtung, Verwerfung und Widerstand als mögliche Formen der Subjektivation (2001: 89).

\section{7 "Herr" und „Knecht": Anerkennungstheoretische Überlegungen}

Die Hegel'sche „Herr-Knecht-Figur“ lässt sich ebenfalls für die vorliegende Problematik fruchtbar machen. Die bisher ausgeführten theoretischen Überlegungen können mit Erläuterungen zur Herr-Knecht-Konzeption sowie zu den davon abgeleiteten Figuren, auf die ich mich in dieser Arbeit beziehe, vervollständigt werden. Die Diskussion dieses theoretischen Bausteins dient insbesondere dazu, die enorme Verkennung des in der Institution Asyl gefangenen Menschen im Verlauf der Inferiorisierung und Zuteilung einer neuen Identität verständlich zu machen.

Der deutsche Philosoph Georg Wilhelm Friedrich Hegel (1770-1831) veröffentlichte im Jahr 1807 sein Hauptwerk Phänomenologie des Geistes unter dem Kanonendonner der Jenaer Schlacht. Die darin enthaltene Dialektik von Herr und Knecht wurde seitdem unzählige Male interpretiert und diskutiert und stellt das wohl berühmteste Unterordnungsverhältnis der westlichen Philosophiegeschichte dar. Das Szenario von Herr und Knecht weist auf die klassische Asymmetrie innerhalb eines Verhältnisses hin, das sich zwischen einem herrischen und einem knechtischen Bewusstsein ergibt (Bertram 2010: 112). In der Herr-Knecht-Dialektik wird aus der systematischen Aufstellung einer Theorie des Selbstbewusstseins die in der dialektischen Dynamik inhärente wechselseitige Anerkennung zwischen Herr und Knecht veranschaulicht (Frigger 2013: 27). 
Hegels Überlegungen zur Entwicklung des Selbstbewusstseins heben die Bedeutsamkeit des Sozialen im Unterschied zur cartesianisch-kantischen Tradition in einer epochalen Art und Weise hervor. Das Selbstbewusstsein ist demnach nicht mehr als eine theoretische, sondern als eine soziale Relation zu verstehen (Bedorf 2011: 74). Die Relation zwischen Ich und dem Anderen stellt sich als dynamische Bewegung dar - als Anerkennung. Es handelt sich um eine radikale Umkehr gegenüber der klassischen Subjektphilosophie, die seit Descartes das Selbstverhältnis als Versenkung des Individuums in seiner Innerlichkeit begreift (Herrmann 2013: 89). Hegel zeigt, dass dieses Selbstverhältnis nicht aus einer autoreflexiven Bewegung - im Sinne des „Cogito ergo sum“ - hervorgeht und nicht aus einem Prozess des Erkennens, sondern vielmehr aus einem solchen des Anerkennens besteht (ebd.). Das Subjekt benötigt „den Anderen“ im Sinne eines anderen Subjekts, um sich selbst zu verwirklichen. Mit den berühmten Hegel-Vorlesungen des russisch-französischen Philosophen Alexandre Kojève (1902-1968), der darin den Begriff der Anerkennung fokussiert, an der Pariser Sorbonne rückte spätestens seit Mitte des 20. Jahrhundert die Frage nach Anerkennung ins Zentrum der Sozialphilosophie (Frigger 2013: 36). Der Mensch ist demnach ein fundamental soziales Wesen. Kojèves Überlegungen fokussieren die intersubjektive Bewegung der Anerkennung. Selbstbewusstsein ist demgemäß weder als Monolith noch als automatischer Prozess zu verstehen, der auch in der Isolation realisiert werden könnte. Vielmehr sind soziale Gemeinschaften und die in ihnen ablaufenden Prozesse aus dieser Sicht von ausschlaggebender Bedeutung für das Subjekt. Ausgangspunkt bildet das Begehren oder die Begierde nach Anerkennung, durch die sich die menschliche von der tierischen Existenz unterscheidet (Kojève 1975: 24). Die Begierde nach Anerkennung bedeutet im Prinzip eine fundamentale Begierde, vom „Anderen“ begehrt zu werden (Herrmann 2013: 91). Das bringt eine entscheidende Wende in der Denkweise mit sich, denn aus dieser Perspektive geht es um die Strukturen des Bewusstseins im Ganzen und eine entsprechend universelle Dialektik, welche die Menschheitsgeschichte neu verstanden zu haben glaubt. Unter dem Einfluss Jean-Paul Sartres und Jacques Lacans stellt sich das HerrKnecht-Motiv immer mehr als ein sozialontologisches Verhältnis dar, das heißt, das Herr-Knecht-Verhältnis ist nicht bloß die Abbildung irgendeiner speziellen Relation, sondern ein allgemeines Muster für soziale Verhältnisse (ebd.: 98). Die sozialontologische Perspektive bezieht sich primär auf die asymmetrische Grundstruktur, die bei Hegel noch symmetrisch ist - Hegels berühmte Formel lautet, „,ie anerkennen sich als wechselseitig anerkennend" (Hegel 1970: 152) - und bei Derrida und Levinas in der Philosophie der Alterität asymmetrisch wird (Bertram 2010: 111). Zentral für den Kontext dieser Studie sind hierbei die folgenden Aspekte, welche die Knecht-Figur und gleichsam den Begriff der Anerkennung, 
der verwendet wird, kennzeichnen (Herrmann 2013: 99 f.): Der erste Gesichtspunkt meint die grundlegende Abhängigkeit des Subjekts von Anerkennung. Das Subjekt kann nicht anders, als nach dieser Anerkennung zu verlangen, weshalb das Selbstbewusstsein im Grunde als „Begierde überhaupt“ zu verstehen sei. Zum zweiten hat diese Abhängigkeit existenziellen Charakter. Sie ist existenziell für die Ausbildung einer Identität im Sinne einer sozialen Existenz. Der dritte Punkt ist der asymmetrische Charakter der Abhängigkeit des Knechts. Dies bezieht sich auf die Asymmetrie des Anerkennungsprozesses, denn der Knecht ist auf gewisse Weise „abhängiger“ als der Herr. Das bringt die Struktur von Herrschaft und Knechtschaft insofern mit sich, als sie Teil einer jeden kommunikativen Beziehung sind (Bertram 2010: 111). Es ist die Wechselseitigkeit von Rede und Antwort, die in dieser Analogie die Knechtschaft und Herrschaft bildet, denn der Kommunikation in Form von Rede und Antwort ist immer das asymmetrische Moment inhärent, das sich in der Knecht-Figur ausdrückt (Herrmann 2013: 99). Wechselseitigkeit bedeutet gleichsam zeitliche Diskontinuität. Für Kommunikations- und damit Anerkennungsprozesse heißt das, dass stets ein Subjekt eine „Vorausleistung“ erbringen muss, um anschließend auf eine Antwort zu warten (ebd.). Dies kann man sich am Beispiel des alltäglichen Grußes vor Augen führen. Ein Subjekt muss den ersten Schritt im Kommunikationsbzw. Anerkennungsprozess wagen, wodurch eine Ungleichheit entsteht. Es ist genau diese Ungleichheit, die Hegel als asymmetrisches Moment von Anerkennung betrachtet und als Figur des „harten Herzens“ (Hegel 1970: 490) illustriert. Das „harte Herz“ des Herrn übergeht die „Vorausleistung“ des Knechts und reflektiert nicht die Anerkennung, die ihm durch ihn zuteilwird (Bertram 2010: 118). Die Ungleichheit auf Seiten des Knechts spiegelt sich in seiner Vulnerabilität wider (Herrmann 2013: 101). Auf der Ebene der Kommunikation entsteht die Verletzbarkeit dadurch, dass das unterlegene Subjekt riskieren muss, keinen Gruß zur Erwiderung zu erhalten. Die Tatsache, dass ein Individuum nicht als anerkennendes anerkannt wird, ist bei Hegel der hauptsächliche Grund des Scheiterns im Akt der Anerkennung (Bertram 2010: 118). Anerkennungskämpfen geht somit eine gescheiterte Anerkennung, eine Verkennung voraus, die das Subjekt angesichts des Verlusts der adäquaten Adressierung durch andere an seiner Existenz zweifeln lässt (Herrmann 2013: 111). Auf die soziale Dimension übertragen, lässt sich die ausbleibende angemessene Anerkennung in Anlehnung an Goffman (vgl. Abschn. 4.1) als sozialer oder bürgerlicher Tod interpretieren. Nicht das physische Überleben ist infrage gestellt, sondern das „In-der-Welt-Sein“ (ebd.: 112). Denn durch den sozialen Ausschluss löst sich das Selbst auf - die Verkennung des Subjekts macht ihn zum „Ding“ (Verdinglichung) (Buck-Morss 2011: 74 f.). Jegliche Selbstgewissheit ist dem Subjekt genommen. Doch bevor 
der Mensch diesen Tod hinnimmt, akzeptiert er freiwillig Demütigung und Unterdrückung, da dies letztlich die grundlegendste Form der Anerkennung darstellt. Auf diese Weise kann das Individuum immer noch an seinem Menschsein festhalten, was als „Paradox der Entmenschlichung“ (Margalit 1996: 137) bezeichnet wird. Aus der Dynamik der Missachtung des Menschen geht demgemäß das paradoxe Verhältnis der verkennenden Anerkennung hervor, das für das Verständnis der in dieser Studie angestellten Überlegungen wichtig ist. Für die vorliegende Diskussion ist vor allem der intersubjektive Aspekt zwischen höhergestelltem und unterlegenem Subjekt von Belang. Die gewählte Denkfigur eignet sich daher sehr gut für die Verwendung innerhalb des Asylkontextes, denn die wirkmächtige Figur deutet auf eine Form der Selbstreferenz hin, die bereits Fremdreferenz und damit soziale Inklusions- und Exklusionsmechanismen impliziert (Otto 2014: 157). Die Termini ,wir“ und ,die Anderen“" stehen ebenfalls in Bezug zum Herr-Knecht-Verhältnis und werden im Rahmen meiner Überlegungen so verstanden, dass das „Wir“ die Eigengruppe, also die dominante Mehrheitsgesellschaft, und „die Anderen“ die Fremdgruppe der Asylbewerber*innen meint. Die Ablehnung des Asylantrags ,der Anderen“ wird als das Scheitern der Anerkennung verstanden, nachdem gleichermaßen buchstäblich so viel riskiert worden ist.

Open Access Dieses Kapitel wird unter der Creative Commons Namensnennung 4.0 International Lizenz (http://creativecommons.org/licenses/by/4.0/deed.de) veröffentlicht, welche die Nutzung, Vervielfältigung, Bearbeitung, Verbreitung und Wiedergabe in jeglichem Medium und Format erlaubt, sofern Sie den/die ursprünglichen Autor(en) und die Quelle ordnungsgemäß nennen, einen Link zur Creative Commons Lizenz beifügen und angeben, ob Änderungen vorgenommen wurden.

Die in diesem Kapitel enthaltenen Bilder und sonstiges Drittmaterial unterliegen ebenfalls der genannten Creative Commons Lizenz, sofern sich aus der Abbildungslegende nichts anderes ergibt. Sofern das betreffende Material nicht unter der genannten Creative Commons Lizenz steht und die betreffende Handlung nicht nach gesetzlichen Vorschriften erlaubt ist, ist für die oben aufgeführten Weiterverwendungen des Materials die Einwilligung des jeweiligen Rechteinhabers einzuholen.

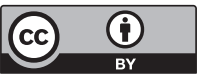

